\title{
Elucidating the contribution of Rayleigh scattering to the bluish appearance of veins
}

Spencer R. Van Leeuwen Gladimir V. G. Baranoski 


\title{
Elucidating the contribution of Rayleigh scattering to the bluish appearance of veins
}

\author{
Spencer R. Van Leeuwen and Gladimir V. G. Baranoski* \\ University of Waterloo, Natural Phenomena Simulation Group, School of Computer Science, Waterloo, Canada
}

\begin{abstract}
The bluish appearance of veins located immediately beneath the skin has long been a topic of interest for biomedical optics researchers. Despite this interest, a thorough identification of the specific optical processes responsible for this phenomenon remains to be achieved. We employ controlled in silico experiments to address this enduring open problem. Our experiments, which are supported by measured data available in the scientific literature, are performed using first-principles models of light interaction with human skin and blood. Using this investigation approach, we quantitatively demonstrate that Rayleigh scattering caused by collagen fibrils present in the papillary dermis, a sublayer of the skin, can play a pivotal role in the bluish appearance of veins as suggested by previous works in this area. Moreover, also taking color perception aspects into account, we systematically assess the effects of variations in fibril radius and papillary dermis thickness on the coloration of veins under different illuminants. Notably, this assessment indicates that Rayleigh scattering elicited by reticulin fibrils, another type of fibril found in the papillary dermis, is unlikely to significantly contribute to the bluish appearance of veins. By strengthening the current understanding of light attenuation mechanisms affecting the appearance of skin and blood, our investigation contributes to the development of more effective technologies aimed at the noninvasive measurement of the physiological properties of these tissues. $\odot$ The Authors. Published by SPIE under a Creative Commons Attribution 3.0 Unported License. Distribution or reproduction of this work in whole or in part requires full attribution of the original publication, including its DOI. [DOI: 10.1117/1.JBO.23.2.025001]
\end{abstract}

Keywords: vein appearance; tissue optics; predictive simulation; skin spectral responses; blood spectral responses; Rayleigh scattering.

Paper 170691PR received Oct. 24, 2017; accepted for publication Jan. 4, 2018; published online Feb. 6, 2018.

\section{Introduction}

Complex optical processes often lead to counter-intuitive material appearances. Such is the case for the appearance of veins located immediately beneath the skin (Fig. 1), usually referred to as subcutaneous veins. While skin and venous blood normally do not appear blue in isolation, their combined spectral responses can result in what a human observer may perceive as a bluish appearance.

One light attenuation process known to elicit blue color in nature is Rayleigh scattering. ${ }^{1,2}$ This type of scattering, caused by structures smaller in magnitude than the wavelength of the affected light, has a stronger effect at the blue end of the visible spectrum. In an early investigation of skin optical properties, it was suggested by Edwards and Duntley ${ }^{3}$ that Rayleigh scattering occurring in the epidermis might be responsible for the bluish appearance of veins. However, Anderson and Parrish observed that since the ratio of diffuse transmittance to direct transmittance in the stratum corneum and epidermis is characterized by weak wavelength dependence, there is no significant amount of Rayleigh scattering in these tissues. Alternatively, they suggested that a form of scattering characterized by a stronger wavelength dependence could be found in the dermis. It is worth noting that Findlay ${ }^{5}$ made a similar observation, although he instead referred to scattering in the dermis as the reflection of light by dermal collagen fibers.

*Address all correspondence to: Gladimir V. G. Baranoski, E-mail: gvgbaran @ cs.uwaterloo.ca
Oettlé ${ }^{6}$ speculated that some form of wavelength-dependent scattering might be caused by fibrils composing the microstructure of collagen fibers found in the dermal tissues of mammals. $\mathrm{He}$ also suggested that an irregularity in size and a lack of parallelism of these collagen fibrils, as can be found in the human dermis, ${ }^{7}$ would not allow for optical interference. Saidi et al. ${ }^{8}$ suggested that the scattering in the dermal tissues could have a contribution from Mie scattering caused by collagen fibers and from Rayleigh scattering caused by smaller tissue structures. Subsequently, Jacques ${ }^{9}$ also speculated that Rayleigh scattering caused by collagen fibrils could explain the good agreement between his mathematical formulation for scattering in the human dermis and measured data.

Cotton and Claridge ${ }^{10}$ stated that the conditions for Rayleigh scattering occurring in the papillary dermis would be met with collagen fibers having a diameter of one order of magnitude smaller than the incident light. Electron microscope measurements performed by Arao et al., ${ }^{7}$ however, show that the collagen fibrils, instead of the fibers, have a diameter with this order of magnitude.

The most comprehensive investigation of the bluish appearance of veins to date, presented by Kienle et al., ${ }^{11}$ suggested that it results from several factors. According to their analysis, blue light does not penetrate as deeply into the skin as red light. Consequently, although blood absorbs blue light more strongly than red, less of the blue light reaches the vein. They suggested that this process, combined with color perception aspects, provides an explanation for the bluish appearance of veins. It is important to take into account, however, the distinct levels of abstraction employed to characterize the target materials, 


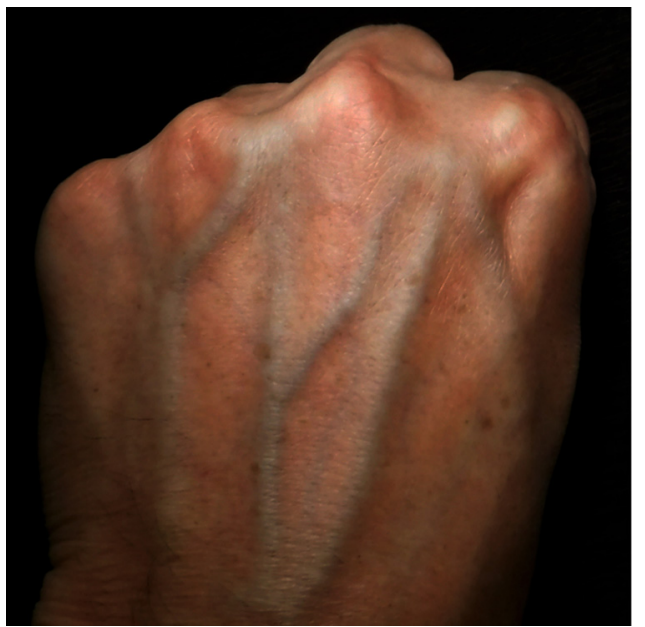

Fig. 1 Photograph illustrating the distinct contrast of skin appearance caused by subcutaneous veins.

notably human skin, in their investigation. For example, in their in silico experiments involving Monte Carlo simulations, skin was represented by a single homogeneous medium. Kienle et al. ${ }^{11}$ stated themselves that a multilayer model of human skin should be used for the complete modeling of their in vivo measurements. Furthermore, in their in vitro experiments, considering five distinct wavelengths and a single illuminant, they employed a phantom medium (a fat emulsion) whose absorptive properties differ markedly from human skin. These experimental constraints might have precluded Kienle et al., ${ }^{11}$ from identifying specific optical processes resulting from the attenuation of light by heterogeneous media (e.g., Rayleigh scattering) or the location of these processes in the skin.

Recently, the suggestion of Rayleigh scattering being elicited by collagen fibrils $6,8,9,10$ was taken into account by Baranoski et al. ${ }^{12}$ during an in silico investigation of the bluish or purple appearance associated with peripheral cyanosis. More specifically, they used measured radius values for collagen fibrils present in the papillary dermis, obtained by Arao et al., ${ }^{7}$ to demonstrate that it is necessary to consider Rayleigh scattering to elicit the characteristic hues of cyanotic skin.

In this paper, which is an updated and extended version of a conference presentation, ${ }^{13}$ we use a similar approach to show that Rayleigh scattering caused by collagen fibrils present in the papillary dermis can play a pivotal role in the bluish appearance of veins. In our investigation, we employ in silico experiments performed using first-principles models of light interaction with human skin and blood. These models, hyperspectral light impingement on skin (HyLIoS) ${ }^{14}$ and cell-based light interaction model for human blood (CLBlood) ${ }^{15,16}$ respectively, account for all significant light-attenuating materials in the skin and blood. The use of an in silico framework allowed for highly controlled and reproducible experiments considering detailed specimen characterizations. It also made it possible to disable the putative Rayleigh scattering occurring in the papillary dermis to assess its effect on the appearance of veins. To the best of our knowledge, the technology required to disable light scattering within a thin skin layer, like the papillary dermis, under in situ conditions is not available. Assuming that one could attempt to perform such a procedure under in vitro conditions, the processes required to extract and prepare a skin sample would alter its optical properties. ${ }^{17}$
It is worth remarking that the word "bluish" is used in scientific literature to describe skin tones that appear subjectively blue. As such, bluish skin tones, such as those caused by veins located immediately beneath the skin, may appear closer to gray than blue. Accordingly, the research findings presented in this paper have a visual component to allow for a qualitative assessment of appearance.

The remainder of this paper is organized as follows. In Sec. 2, we discuss light attenuation processes that occur within the skin and blood in the visible domain (400 to $700 \mathrm{~nm}$ ). This will serve as a foundation for our investigation and discussion. Next, in Sec. 3, we concisely describe the models employed in this investigation and how they were employed to simulate the optical properties of a skin specimen with a vein immediately beneath it. We then outline our in silico experimental setup in Sec. 4, present our findings in Sec. 5, and discuss their implications in Sec. 6. In Sec. 7, we conclude the paper and provide potential directions for future work.

\section{Biophysical Background}

In this section, we describe the tissues relevant to the appearance of veins. For each tissue, we outline the constituent materials that contribute significantly to the attenuation of light in the visible domain. Measured absorption spectra for all pigments discussed in this section are presented in Fig. 2. We also discuss the role of the detour and sieve effects in the optical properties of skin and blood.

\subsection{Skin}

The cutaneous tissues are usually divided into three distinct layers, namely the stratum corneum, epidermis, and dermis, from the outermost to the innermost. ${ }^{24,25}$ The epidermis can be further subdivided into four sublayers: stratum lucidum, stratum granulosum, stratum spinosum, and stratum basale. The dermis, in turn, can be subdivided into two sublayers: papillary dermis and reticular dermis. It is worth noting that the stratum lucidum is a clear layer found only in thick skin regions, such as the palms and soles. ${ }^{24}$ For this reason, it is usually not considered in skin optics investigations.

The absorption of light traversing the stratum corneum and epidermis is dominated by melanin with a minor contribution from beta-carotene [Fig. 2(b)]. ${ }^{25}$ Melanin is synthesized and preferentially concentrated in the stratum basale. ${ }^{24}$ Melanin can be classified as either brown-black eumelanin or yellowred pheomelanin [Fig. 2(a)], the former of which has a significantly higher concentration in skin. Melanin can be dispersed throughout the epidermis in colloidal form or clustered within organelles called melanosomes. ${ }^{26}$

In the skin, light is mostly scattered by heterogeneous structures, such as cells, organelles, and fibers. ${ }^{25}$ Goniometric measured $\operatorname{data}^{27}$ and empirical observations available in the literature ${ }^{4,28}$ indicate that both the stratum corneum and epidermis are characterized by a strong forward scattering behavior exhibiting slight variations with wavelength. Scattering in the epidermis is dominated by melanosomes, ${ }^{29}$ which can be described as particles with the shape of a prolate spheroid ${ }^{30}$ and a melanin concentration of $17.9 \%$ to $72.4 \% .{ }^{29}$ In lightly pigmented specimens, melanosomes can occur in groups surrounded by a transparent membrane known as melanosome complexes. ${ }^{30,31}$ In addition to empirical observations indicating that a significant amount of Rayleigh scattering does not occur in the stratum corneum and epidermis, ${ }^{4,5}$ it has been shown that 


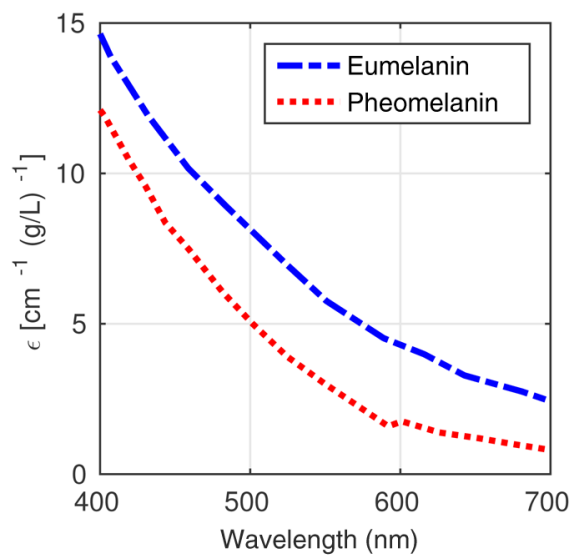

(a)

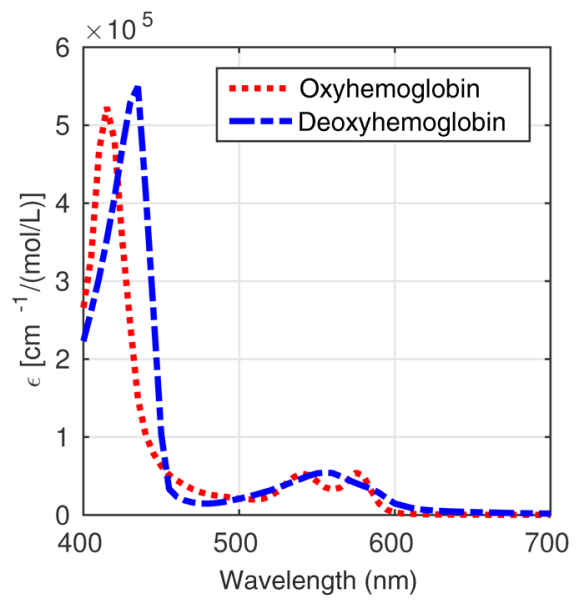

(c)

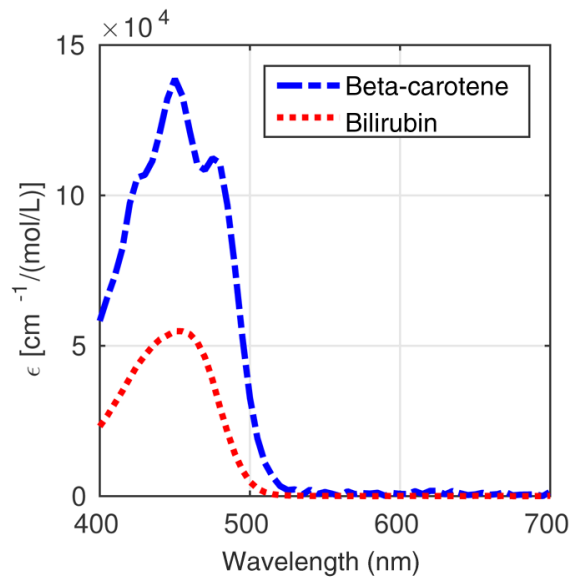

(b)

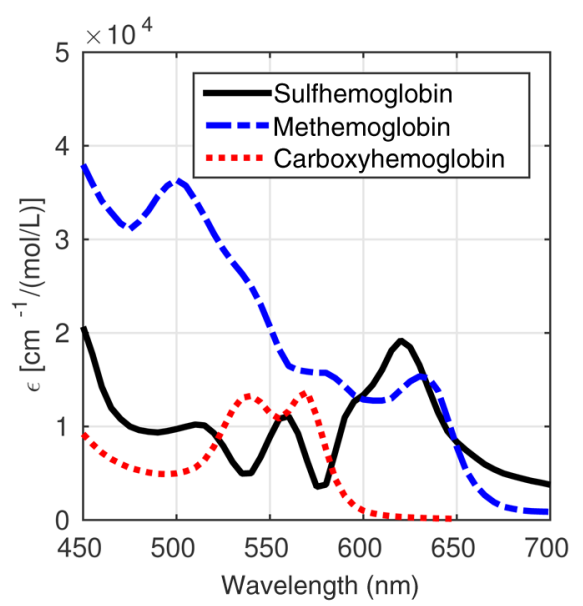

(d)

Fig. 2 Absorption spectra for skin and blood pigments with a significant impact on light attenuation in the visible domain. (a) Extinction coefficient curves for the melanins considered in this investigation. ${ }^{18}$ (b) Molar extinction coefficient curves for beta-carotene and bilirubin. ${ }^{19}$ (c) Molar extinction coefficient curves for the functional hemoglobins. ${ }^{20}$ (d) Molar extinction coefficient curves for the dysfunctional hemoglobins. ${ }^{21-23}$

the consideration of scattering caused by melanosomes and melanosome complexes is sufficient to predictively model the scattering behavior of the stratum corneum and epidermis in the blue region of the light spectrum. ${ }^{14}$

The outermost dermal layer, the papillary dermis, is composed of loosely packed collagen and reticulin fibers, which, in turn, are composed of smaller-sized fibrils. Since these fibrils are smaller in magnitude than the wavelength of light within the visible spectrum, any scattering caused by these structures would likely follow the Rayleigh scattering theory. ${ }^{8}$ The innermost dermal layer, the reticular dermis, is composed of dense irregular connective tissue formed by densely packed collagenous fibers arranged in a tight interwoven pattern. ${ }^{24}$ A network of blood vessels also runs throughout the dermis with wider vessels in the reticular dermis. Blood-borne pigments within these vessels [Figs. 2(b)-2(d)] dominate the dermis's absorptive behavior in the visible domain. ${ }^{32}$ Furthermore, the diffusion of red light traversing the reticular dermis ${ }^{33}$ results from scattering by the dense collagen fibers (following a Mie scattering behavior $^{9}$ ) and the wider blood vessels. Since this scattering is significant enough to diffuse the light, ${ }^{33}$ it would likely dominate any Rayleigh scattering that may occur in the reticular dermis.

\subsection{Blood}

This highly specialized connective tissue is composed of plasma and various types of cells known as formed elements. ${ }^{34}$ Red blood cells, or erythrocytes, make up $99 \%$ of the formed elements in the blood, and each one consists of a thin membrane encapsulating a hemoglobin solution. The attenuation of light by red blood cells exceeds that of other blood components by 2 to 3 orders of magnitude. ${ }^{34}$ The absorption of light by red blood cells in the visible domain is primarily attributed to the hemoglobins [Figs. 2(c) and 2(d)], and the scattering of light is determined by their shape and orientation. ${ }^{35}$

The orientation of red blood cells in a flowing blood sample can change depending on its shear rate (i.e., the velocity gradient in the direction normal to the flow). ${ }^{36}$ At low, intermediate, and high shear rates, the red blood cells are predominantly randomly oriented, rolling, or aligned with the direction of the flow, respectively. ${ }^{36}$ When the volume fraction of blood occupied 
by formed elements, known as hematocrit, is above $40 \%$, the alignment of the red blood cells becomes more pronounced.

\subsection{Hypodermis}

This subcutaneous tissue is located immediately beneath the skin except for certain regions of the body (e.g., eyelids) in which it is not present. ${ }^{24}$ It consists mostly of white fat cells that contain lipidic vesicles. ${ }^{37}$ The size of these spherical droplets of lipids is larger than that of typical scatterers. ${ }^{38}$ This may explain the strong backscattering behavior of this adipose tissue, comparable with the dermis's behavior and much stronger than the behavior of other types of tissues like cartilages and muscles, which has been verified in actual experiments. ${ }^{39}$ The most abundant light absorbers found in the hypodermis, namely water $\left(10 \%\right.$ to $\left.30 \%^{37}\right)$ and lipids $\left(70 \%\right.$ to $\left.90 \%^{37}\right)$, have a negligible impact in the visible domain. ${ }^{40-43}$ Due to the strong absorptive properties of cutaneous melanin in the blue region of the light spectrum, only a negligible amount of light in this region is likely to reach the hypodermis. ${ }^{25}$ Experiments by Dinish et al. ${ }^{44}$ show that $90 \%$ to $95 \%$ of light in the red and green regions of the light spectrum are reflected by white adipose tissue. Accordingly, the majority of the visible light that reaches the hypodermis is likely to be scattered by the droplets of lipids and remitted into the reticular dermis. ${ }^{45}$

\subsection{Vein Wall}

Vein walls are composed of elastin, collagen, and muscle tissue. ${ }^{46}$ The absorption of visible light within a vein wall is dominated by hemoglobin in the muscle tissue [Figs. 2(c) and $2(\mathrm{~d})] .{ }^{47}$ It is also possible that light traversing a vein wall may be scattered by its heterogeneous structures.

\subsection{Detour and Sieve Effects}

Skin and blood are both turbid mediums characterized by their heterogeneously distributed, pigment-containing structures. Notably, the attenuating properties of melanosomes and red blood cells play an important role in the optical processes of skin and blood, respectively. When light traverses a turbid medium, scattering by these structures can increase its optical pathlength, which results in an increased probability of absorption. Conversely, light may not encounter any pigment-containing structure and, therefore, has a minimal chance of absorption. These phenomena are, respectively, known as the detour effect ${ }^{48}$ and the sieve effect. ${ }^{49}$ The combined contribution of these effects to the overall absorptive behavior of a turbid medium depends on the properties and distribution of the medium's pigment-containing structures.

\section{Experimental Framework}

\subsection{Model Background and Setup}

In our in silico experiments, we use HyLIoS ${ }^{14}$ and CLBlood ${ }^{15,16}$ to simulate the interactions of light with skin and flowing venous blood, respectively. Both of these models have been evaluated extensively against measured data in their original publications. ${ }^{14,15}$ CLBlood was also recently updated and reevaluated. $^{16}$

HyLIoS and CLBlood are both first-principles models that employ measured optical properties of constituent materials to control the propagation and attenuation of light within human skin and blood. Although this paper is focused on light in the visible domain, it is worth noting that both models consider all constituent materials with a significant contribution to light attenuation in the ultraviolet, visible, and near-infrared domains. Measured optical properties for these constituent materials were obtained from scientific literature and made available online. ${ }^{50,51}$ References to this data can also be found in the models' original publications. ${ }^{14,15}$ Additionally, HyLIoS and CLBlood account for sieve and detour effects by individually distributing melanosomes throughout the epidermis and red blood cells throughout the plasma, respectively. This includes the grouping of melanosomes within melanosome complexes. As pointed out by Lister et al., ${ }^{17}$ the size and distribution of these melanin-containing structures have been often overlooked when simulating their effects on skin color.

In our experiments, we computed modeled reflectance data for skin specimens with and without a vein immediately beneath the skin. Henceforth, we will refer to these specimens as skin specimens with and without a subcutaneous vein. The representation of these specimens in our modeling framework is described in the following sections.

\subsection{Skin Specimen without a Subcutaneous Vein}

For the skin specimens without a subcutaneous vein, we used the HyLIoS model, as described in its original publication. ${ }^{14}$ In particular, there is a layer below the reticular dermis that represents the hypodermis [Fig. 3(a)]. We remark, as outlined in Sec. 2.3, that the amount of light in the blue region reaching this layer is negligible due to the cutaneous pigmentation ${ }^{25}$ and most of the light in the green and red regions is reflected back. $^{44}$ Thus, by reflecting the visible light that may reach the hypodermis, a strategy also employed by several relevant works on skin color reproduction (e.g., the interested reader is referred to the works by Cotton and Claridge ${ }^{10}$ and by Doi and Tominaga ${ }^{52}$ ), one can minimize the introduction of undue computational overhead in the simulations without reducing the fidelity of the simulations. Accordingly, we assume that visible light reaching the hypodermis is diffusely reflected back into the reticular dermis. The diffusion distribution in this case accounts for the overall scattering behaviors of both reticular dermis ${ }^{33}$ and hypodermis, ${ }^{39}$ as also outlined earlier (Secs. 2.1 and 2.3). The implications of this simulation choice are further discussed in Sec. 6.5.

\subsection{Skin Specimen with a Subcutaneous Vein}

For the skin specimens with a subcutaneous vein, the hypodermis layer is replaced by the vein [Fig. 3(b)]. We use a three layer model to represent the vein [Fig. 3(b)]. The first and third layers represent the vein wall and the second layer employs CLBlood to account for flowing venous blood. When light encounters the boundary of a layer, a Fresnel test ${ }^{25}$ is performed to determine whether the light is reflected or transmitted.

We do not consider any absorption or scattering of light within the vein wall. In addition, any light that is transmitted below the bottom vein wall layer is discarded. The scientific reasoning behind each of these simulation choices, which were also implemented to avoid undue computational overhead without reducing the fidelity of our simulations, is further discussed in Secs. 6.1 and 6.6, respectively. 


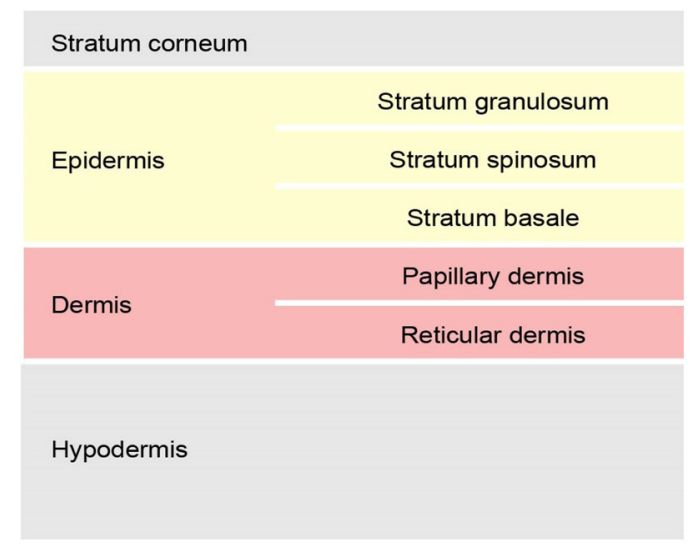

(a)

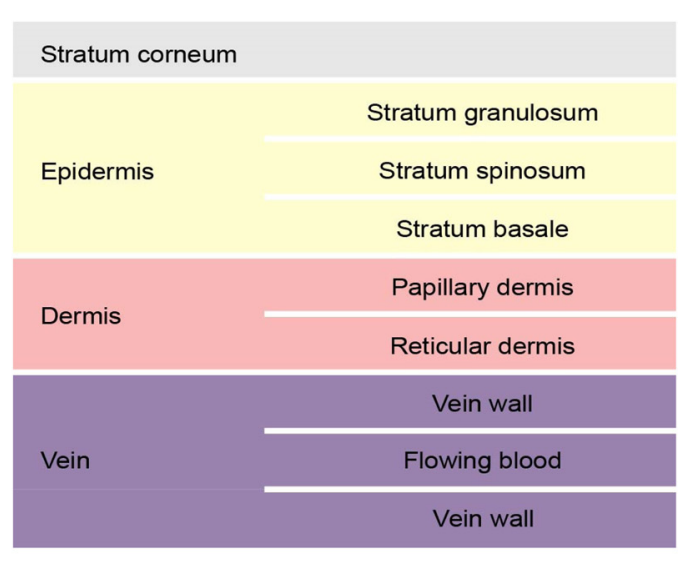

(b)

Fig. 3 Diagrams depicting the layers considered in our experimental framework for skin specimens: (a) without a subcutaneous vein and (b) with a subcutaneous vein.

\subsection{Vein Precomputation}

Since we know that visible light transmitted by the dermis is diffusely distributed when considering a typical physiological thickness, ${ }^{33}$ we can assume a uniform distribution for the angle of incidence of all light that encounters the vein. This assumption allows us to precompute the probability that light is reflected by the vein using the representation outlined in Sec. 3.3. To obtain this probability, we first compute the reflectance of the vein considering polar angles in the range [0 deg, $90 \mathrm{deg}$ ) at each 5-deg interval and azimuthal angles in the range [0 deg, $360 \mathrm{deg}$ ) at each 90-deg interval. For each angle of incidence, the reflectance calculations are performed considering $10^{5}$ sample rays per wavelength. Since we assume that the light entering the vein is uniformly distributed, we average the reflectance values across all samples at each wavelength to get the overall probability of light being reflected by the vein. When performing our experiments, we employ this precomputed probability to determine whether a light ray that encounters the vein will be reflected diffusely or discarded (i.e., absorbed or transmitted).

\section{In Silico Experimental Setup}

In our experiments, we computed directional-hemispherical reflectance curves for various skin specimens with a virtual spectrophotometer ${ }^{53}$ considering a 5-deg angle of incidence, $10^{6}$ sample rays per wavelength, and a 5-nm spectral resolution. We then used the computed reflectance data to generate textured color swatches using the method described in Sec. 4.4.

In each experiment set, we consider skin specimens with and without a subcutaneous vein. The skin specimen characterizations considered in each experiment set are outlined in Sec. 4.1. We remark that they are based on measured data provided in the scientific literature. To evaluate the contribution of Rayleigh scattering to the reflectance and appearance of the specimens, each experiment set includes results that consider Rayleigh scattering as well as results that do not consider it. The method used to disable Rayleigh scattering occurring in the papillary dermis is presented in Sec. 4.3. The vein-related parameters and quantities considered in all experiments are described in Sec. 4.2, and the online reproducibility of the results presented in this paper is addressed in Sec. 4.5.

\subsection{Experimental Sets}

We performed three sets of in silico experiments. In experiment set \#1 (Figs. 6 and 7), we consider a baseline skin specimen characterized by the parameter values presented in Tables 1 and 2. Accompanying each of these values is a list of references that provide their physiological basis. We remark that the thickness of our skin specimen is within a measured range of thickness for skin on the dorsum of the hand. ${ }^{54}$

In experiment set \#2 (Fig. 8), we multiply the melanin content and dermal blood content parameters in Table 2 by $f_{\text {mel }} \in\{1.0,1.5,2.0,2.5\}$ and $f_{\mathrm{bl}} \in\{1.0,1.5,2.0,2.5\}$, respectively, to obtain additional skin specimens. For each experiment, the mean epidermal melanin content is in the range $1 \%$ to $3 \%$ and dermal blood content is in the range $2 \%$ to $6 \%$, corresponding to the physiological characterization of a lightly pigmented specimen ${ }^{64}$ Our justification for focusing on lightly pigmented specimens in our investigation is provided in Sec. 6.3.

In experiment set \#3 (Figs. 9-13), we varied the radius of the fibrils in the papillary dermis and the thickness of the papillary dermis. We considered fibril radius values in the range 20 to $60 \mathrm{~nm}$ and papillary dermis thickness values in the range from 0.0025 to $0.02 \mathrm{~cm}$. These ranges are based on measurements of collagen and reticulin fibril radius ${ }^{7,74}$ as well as reported values for papillary dermis thickness., ${ }^{4,63,74,91}$

\subsection{Vein-Related Data}

In our precomputation (Sec. 3.4) of the probability of light being reflected by the vein $\left[p_{\text {vein }}^{r}(\lambda)\right]$, we considered the blood characterization data presented in Table 3 . It is worth noting that the thickness of the blood sample, $1270 \mu \mathrm{m}$, corresponds to the lumen diameter of a vein on the dorsum of the hand. ${ }^{94}$ The refractive index selected for the vein wall is 1.39 , which corresponds to a measured value for the vena femoralis. ${ }^{99}$ The resulting curve for $p_{\text {vein }}^{r}(\lambda)$ is presented in Fig. 4.

\subsection{Rayleigh Scattering Simulation}

Within the HyLIoS' formulation, ${ }^{14}$ the direction of a light ray traversing the papillary dermis can be perturbed considering the Rayleigh scattering distribution. ${ }^{100}$ Ideally, one would like to consider the exact geometric shape of the fibrils and directly simulate the interaction of light with each one of them. However, 
Table 1 HyLloS parameters employed in the general characterization of all skin specimens considered in this investigation. Note that, in the last two rows, the scatterers correspond to the fibrils in the papillary dermis.

\begin{tabular}{|c|c|c|}
\hline HyLloS parameters & Values & References \\
\hline Aspect ratio of skin surface fold & 0.1 & $54,55,56$ \\
\hline Stratum corneum thickness $(\mathrm{cm})$ & 0.0004 & $54,57-60$ \\
\hline Stratum granulosum thickness $(\mathrm{cm})$ & 0.0033 & 54,61 \\
\hline Stratum spinosum thickness $(\mathrm{cm})$ & 0.0033 & 54,61 \\
\hline Stratum basale thickness $(\mathrm{cm})$ & 0.0033 & 54,62 \\
\hline Reticular dermis thickness (cm) & 0.125 & $4,54,63$ \\
\hline $\begin{array}{l}\text { Stratum granulosum melanosome } \\
\text { content }(\%)\end{array}$ & 0.0 & 29,64 \\
\hline $\begin{array}{l}\text { Stratum spinosum melanosome } \\
\text { content }(\%)\end{array}$ & 0.0 & 29,64 \\
\hline $\begin{array}{l}\text { Stratum basale melanosome dimensions } \\
(\mu \mathrm{m} \times \mu \mathrm{m})\end{array}$ & $0.41 \times 0.17$ & 30 \\
\hline $\begin{array}{l}\text { Melanosome eumelanin concentration } \\
(\mathrm{mg} / \mathrm{mL})\end{array}$ & 32.0 & 65,66 \\
\hline $\begin{array}{l}\text { Melanosome pheomelanin concentration } \\
(\mathrm{mg} / \mathrm{mL})\end{array}$ & 2.0 & 65,66 \\
\hline Dermal oxyhemoglobin fraction (\%) & 90.0 & 67 \\
\hline $\begin{array}{l}\text { Hemoglobin concentration in blood } \\
(\mathrm{mg} / \mathrm{mL})\end{array}$ & 147.0 & 68,69 \\
\hline $\begin{array}{l}\text { Methemoglobin concentration in blood } \\
(\mathrm{mg} / \mathrm{mL})\end{array}$ & 1.5 & 70 \\
\hline $\begin{array}{l}\text { Carboxyhemoglobin concentration in } \\
\text { blood }(\mathrm{mg} / \mathrm{mL})\end{array}$ & 1.5 & 71 \\
\hline $\begin{array}{l}\text { Sulfhemoglobin concentration in blood } \\
(\mathrm{mg} / \mathrm{mL})\end{array}$ & 0.0 & 23 \\
\hline Blood bilirubin concentration $(\mathrm{mg} / \mathrm{mL})$ & 0.003 & 72 \\
\hline $\begin{array}{l}\text { Stratum corneum beta-carotene } \\
\text { concentration }(\mathrm{mg} / \mathrm{mL})\end{array}$ & $2.1 \times 10^{-4}$ & 73 \\
\hline $\begin{array}{l}\text { Epidermis beta-carotene concentration } \\
(\mathrm{mg} / \mathrm{mL})\end{array}$ & $2.1 \times 10^{-4}$ & 73 \\
\hline $\begin{array}{l}\text { Blood beta-carotene concentration } \\
(\mathrm{mg} / \mathrm{mL})\end{array}$ & $7.0 \times 10^{-5}$ & 73 \\
\hline Stratum corneum water content (\%) & 35.0 & 74,75 \\
\hline Epidermis water content (\%) & 60.0 & 74,76 \\
\hline Papillary dermis water content (\%) & 75.0 & 74,76 \\
\hline Reticular dermis water content (\%) & 75.0 & 74,76 \\
\hline Stratum corneum lipid content (\%) & 20.0 & 77 \\
\hline Epidermis lipid content (\%) & 15.1 & $74,78,79$ \\
\hline Papillary dermis lipid content (\%) & 17.33 & $74,78,79$ \\
\hline
\end{tabular}

Table 1 (Continued).

\begin{tabular}{lcc} 
HyLloS parameters & Values & References \\
\hline Reticular dermis lipid content (\%) & 17.33 & $74,78,79$ \\
Stratum corneum keratin content (\%) & 65.0 & $62,80,81$ \\
$\begin{array}{l}\text { Stratum corneum urocanic acid density } \\
\text { (mol/L) }\end{array}$ & 0.01 & 82 \\
Skin DNA density (mg/mL) & 0.185 & $74,83,84$ \\
Stratum corneum refractive index & 1.55 & 85,86 \\
Epidermis refractive index & 1.4 & 85,87 \\
Papillary dermis refractive index & 1.39 & 33,85 \\
Reticular dermis refractive index & 1.41 & 33,85 \\
Melanin refractive index & 1.7 & 88 \\
$\begin{array}{l}\text { Papillary dermis scatterers' refractive } \\
\text { index }\end{array}$ & 1.53 & 89 \\
$\begin{array}{l}\text { Papillary dermis fraction occupied by } \\
\text { scatterers (\%) }\end{array}$ & 22.0 & 9 \\
\hline
\end{tabular}

such an approach not only would represent a challenging project in itself, deserving a publication in its own right like the work of Arifler et al. ${ }^{101}$ on ocular stroma, but also would be severely constrained by data scarcity. Alternatively, one needs to resort to an approximation and take into account its practical implications.

In the work by Saidi et al., ${ }^{8}$ the authors employed infinitely long cylinders placed parallel to the skin surface to represent fibers eliciting Mie scattering. Incidentally, their algorithm was based on the work by Bohren and Huffmann. ${ }^{102}$ Although a similar approximation could be potentially employed for the

Table 2 HyLlos melanin and blood content parameters that are modified to produce different skin specimen characterizations. The presented values correspond to the baseline skin specimen.

\begin{tabular}{|c|c|c|}
\hline HyLloS parameters & Values & s References \\
\hline Stratum basale melanosome content (\%) & $1.0^{*}$ & 29,64 \\
\hline $\begin{array}{l}\text { Stratum granulosum colloidal melanin } \\
\text { content }(\%)\end{array}$ & 0.5 * & $26,29,64,90$ \\
\hline Stratum spinosum colloidal melanin content (\%) & 0.5 * & $26,29,64,90$ \\
\hline Stratum basale colloidal melanin content (\%) & 0.5 * & $26,29,64,90$ \\
\hline Papillary dermis blood content (\%) & $0.2^{\dagger}$ & $9,64,63,69$ \\
\hline Reticular dermis blood content (\%) & $0.2^{\dagger}$ & $9,64,63,69$ \\
\hline Radius of papillary dermis scatterers $(\mathrm{nm})$ & 40.0 & 7,74 \\
\hline Papillary dermis thickness $(\mathrm{cm})$ & 0.02 & $4,54,63,74,91$ \\
\hline
\end{tabular}

Note: The melanin parameters ${ }^{\star}$ and blood parameters ${ }^{\dagger}$ are multiplied by a factor of $f_{\text {mel }}$ and $f_{\mathrm{bl}}$, respectively, to provide the skin specimens in experiment set \#2. 
Table 3 CLBlood parameters employed in the characterization of the blood flowing through the subcutaneous vein.

\begin{tabular}{lcc} 
CLBlood parameters & Values & References \\
\hline Hematocrit $(\%)$ & 45 & 92,93 \\
Sample thickness $(\mu \mathrm{m})$ & 1270.0 & 94 \\
Randomly oriented cells (\%) & 10 & $36,95,96$ \\
Rolling oriented cells (\%) & 60 & $36,95,96$ \\
Aligned oriented cells (\%) & 30 & $36,95,96$ \\
Oxyhemoglobin (\%) & 67.9 & 97 \\
Deoxyhemoglobin (\%) & 29.1 & 97 \\
Sulfhemoglobin (\%) & 0 & 23 \\
Methemoglobin (\%) & 1.5 & 98 \\
Carboxyhemoglobin (\%) & 1.5 & 71 \\
Mean cell hemoglobin content (g/L) & 330.0 & 92 \\
\hline
\end{tabular}

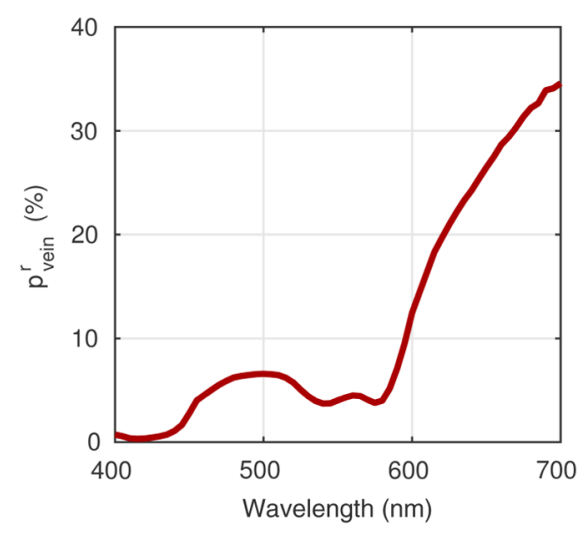

Fig. 4 The precomputed probability of light being reflected by the subcutaneous vein specimen $\left(p_{\text {vein }}^{r}\right)$ employed in our experiments. This curve was computed using the procedure outlined in Sec. 3.4, considering the blood characterization data presented in Table 3.

scattering elicited by fibrils, a few aspects have to be taken into account when assessing its suitability for the problem at hand.

As pointed out by Bohren, ${ }^{103}$ scattering by spheres and by infinitely long circular cylinders illuminated normally to their axes are in some ways similar. For example, spherical Bessel functions employed in the formulation of sphere scattering coefficients correspond to cylindrical Bessel functions employed in the formulation of cylinder scattering coefficients. Different from a sphere, however, an infinitely long cylinder cannot be confined within a finite volume, which impacts the way the scattering varies with the radius of the scatterers. ${ }^{103}$

Moreover, recall that Rayleigh scattering is dependent on the wavelength of light to the power of -4 , which corresponds to the Rayleigh limit of the Mie scattering theory, ${ }^{9,100}$ and it is characterized by forward and backward lobes leading to an isotropic behavior on average. ${ }^{8,100}$ As also pointed out by Bohren, ${ }^{103}$ the use of other type of approximations (e.g., cylinders) in scattering formulations originally proposed considering homogeneous spheres, like the Rayleigh theory, which is included in the Mie theory, would be "risky," especially for computing scattering toward the backward direction.

If the fibrils present in the papillary dermis were characterized by a preferred orientation, this might represent an argument in favor of a cylindrical approximation. However, the irregular distribution of collagen fibrils suggested by Oettlé ${ }^{6}$ was corroborated by the electron microscope images provided by Arao et al., ${ }^{7}$ showing no preferred orientation for these structures in the papillary dermis.

Hence, representing the scatterers of interest, namely the fibrils, by spheres, albeit having the shortcomings of any approximation, provides the best fidelity/cost ratio in comparison with other alternatives, like cylinders, since it allows us to avoid undue complexity and the introduction of bias in the simulation of backscattering, as indicated by Bohren. ${ }^{103}$ For these reasons, we have selected the following formula for the scattering coefficient controlling the probability of Rayleigh scattering events: ${ }^{14,100,104}$

$\mu_{s}^{R}(\lambda)=\frac{32 \pi^{4} r^{3} v_{f}}{\lambda^{4}}\left(\frac{\eta^{2}-1}{\eta^{2}+1}\right)^{2}$,

where $v_{f}$ represents the volume fraction of the papillary dermis occupied by fibrils and $r$ represents the radius of the fibrils. The variable $\eta$ is the ratio between the refractive index of the fibrils $\left(\eta_{\mathrm{f}}\right)$ and the refractive index of the papillary dermis $\left(\eta_{\mathrm{pd}}\right)$.

To deactivate Rayleigh scattering, we simply set $\eta_{\mathrm{f}}=\eta_{\mathrm{pd}}$, which results in $\mu_{s}^{R}=0$ and, hence, a $0 \%$ probability that a Rayleigh scattering event occurs. The values for $v_{\mathrm{f}}, \eta_{\mathrm{pd}}$, and the baseline $\eta_{\mathrm{f}}$ are given in Table 1 . The baseline value for $r$ is given in Table 2 .

It is worth noting that the thickness of the papillary dermis $\left(t_{\mathrm{pd}}\right)$ also indirectly affects how much Rayleigh scattering will occur. This can be explained by the fact that the probability of light being attenuated while traversing a given layer tends to be higher for thicker layers. ${ }^{105}$

\subsection{Swatch Generation}

Swatches are used in this work to approximate the appearance of a given skin specimen under distinct illumination conditions. To generate the color of a swatch, the skin specimen's modeled reflectance curve is convolved with the selected illuminant's relative spectral power distribution and the broad spectral response of the human photoreceptors. ${ }^{106}$ The last step involves a standard CIE XYZ to sRGB conversion procedure, ${ }^{107}$ which includes a white balancing calculation considering the selected illuminant.

In our investigation, we considered four standard CIE (Commission Internationale de l'Eclairage) illuminants, namely D50, D65, A, and F2, ${ }^{106}$ whose relative spectral power distributions are presented in Fig. 5. D50 and D65 approximate daylight at different times of day, A corresponds to an incandescent light, and F2 represents a "cool white fluorescent" light typical of an office environment. ${ }^{106}$ Since F2 was published independently of the other illuminants and the employed data were provided in relative units, we decided to increase its intensity in the swatch generation process to get a similar qualitative brightness across the swatches. We selected D65 for the experimental instances in which we considered a single illuminant (Figs. 8 and 13).

After computing the color of a swatch, we apply a grayscale texture to it to make the synthetic depiction of skin more 


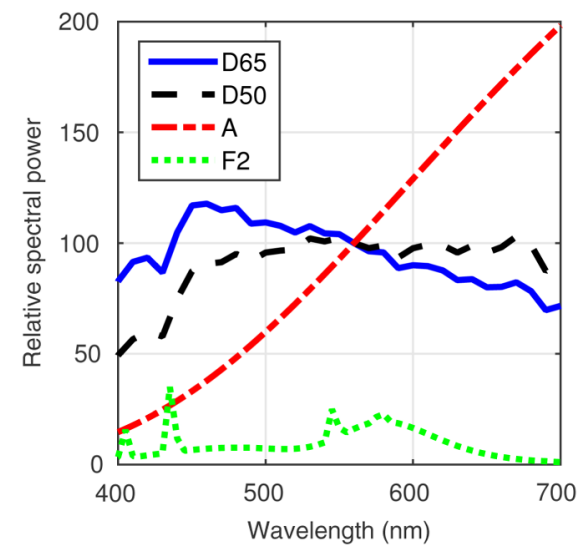

Fig. 5 Relative spectral power distribution of each CIE standard illuminant ${ }^{106}$ employed in the swatch generation process described in Sec. 4.4.

realistic. In Figs. 8 and 13, each swatch depicts a zoomed-out view of skin with a vein running beneath it. Note that, in these swatches, not only the skin area above the vein but also the area around it are rendered with their corresponding colors. This allows us to implicitly account for color perception phenomena related to the retinex theory. ${ }^{108}$ This theory proposes that the spectral remission from a given area alone does not determine its color, i.e., one must also consider the remission from surrounding areas. According to Kienle et al., ${ }^{11}$ retinex theory should be taken into account when assessing the perception of a vein's appearance as seen by a human observer.

\subsection{Online Reproducibility}

The experiments in this paper can be reproduced with our online versions of HyLIoS ${ }^{109}$ and CLBlood, ${ }^{110}$ which are deployed using a custom model distribution system. ${ }^{11,112}$ This system allows a user to perform their own experiments with a specified skin specimen characterization and experimental setup. The online version of HyLIoS has been updated to facilitate the reproduction of the reflectance of a skin specimen with a subcutaneous vein considering a chosen venous blood oxygenation. Additionally, we have made it possible to reproduce the reflectance of an isolated vein from a single angle of incidence using our online version of CLBlood. ${ }^{110}$

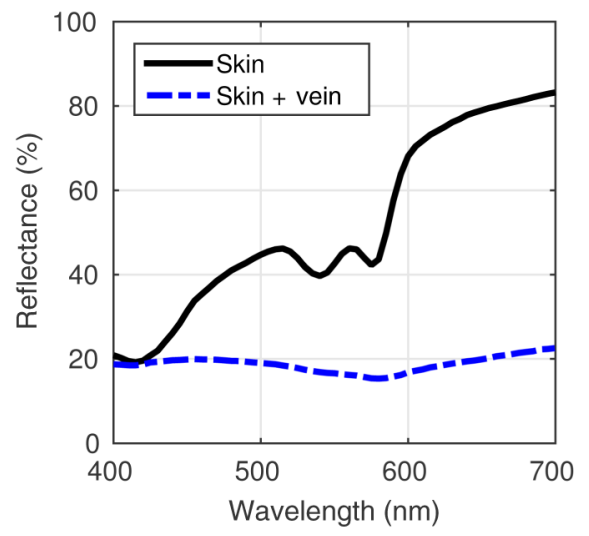

(a)

\section{Results}

\subsection{Experiment Set \#1}

For our first set of experimental results, we explore the effect of disabling Rayleigh scattering in the papillary dermis on the reflectance and appearance of the baseline skin specimen with and without a subcutaneous vein. First, we examine the reflectance results, which are presented in Fig. 6. When Rayleigh scattering is enabled [Fig. 6(a)], there is a smaller difference between the reflectance curves of the skin specimens with and without a subcutaneous vein in the short (blue) wavelength range. In particular, the difference between the two curves is negligible in the region near $400 \mathrm{~nm}$. Given the near zero probability of light being reflected by the vein in this region (Fig. 4), the light must have been scattered out of the skin before it could reach the vein.

Since this behavior could potentially be attributed to Rayleigh scattering, let us examine the reflectance results for the experiments without Rayleigh scattering presented in Fig. 6(b). We can observe that disabling Rayleigh scattering reduces the reflectance significantly for both specimens in the region near $400 \mathrm{~nm}$. Furthermore, the amount of reflected light in this region is lower for the skin specimen with a subcutaneous vein. This indicates that the absence of Rayleigh scattering allowed more light to reach and, hence, be absorbed by the vein.

Now, let us examine Fig. 7 to see how these differences in reflectance translate to appearance. The appearance swatches presented in Fig. 7(a) were generated using the reflectance data from the experiments with Rayleigh scattering [Fig. 6(a)] and considering several illuminants. The colors depicted in these swatches generally match our expectations for the appearance of skin with and without a subcutaneous vein. Accordingly, the RGB (red, green, blue) color triplets used to generate these swatches, presented in Table 4, quantitatively show that the colors elicited by the skin specimen with a subcutaneous vein have a blue component that exceeds in magnitude its red and green counterparts (for all considered illuminants). Conversely, the swatches presented in Fig. 7(b), computed considering the reflectance data from the experiments without Rayleigh scattering [Fig. 6(b)], present a brownish-red appearance for the skin specimen with a subcutaneous vein. For this specimen, we can also observe that the red component of the RGB triplets

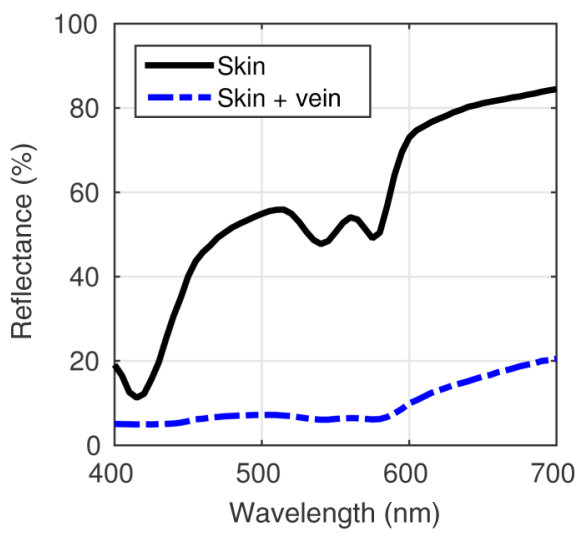

(b)

Fig. 6 Modelled reflectance curves obtained for the baseline skin specimen with and without a subcutaneous vein: (a) with Rayleigh scattering and (b) without Rayleigh scattering. 


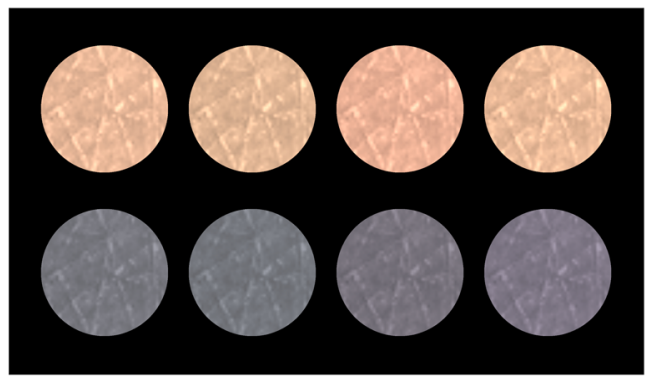

(a)

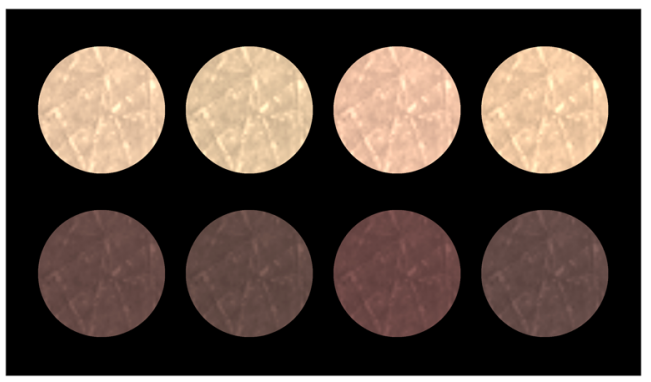

(b)

Fig. 7 Generated swatches depicting the effect of Rayleigh scattering in the papillary dermis on the appearance of the baseline skin specimen without (top) and with (bottom) a subcutaneous vein. From left to right, the swatches in each subfigure were generated considering CIE standard illuminants D50, D65, A, and F2: (a) with Rayleigh scattering and (b) without Rayleigh scattering.

Table 4 With Rayleigh scattering, the RGB (red, green, blue) triplets employed in the generation of the swatches presented in Fig. 7. Each RGB triplet is computed using the modelled reflectance data for the baseline skin specimen with and without a subcutaneous vein considering $\mathrm{ClE}$ standard illuminants D50, D65, A, and F2, as described in Sec. 4.4.

D50

$(242,191,159)$

$(124,125,133)$
D65

$(234,189,155)$

$(121,124,131)$
A

$(256,185,156)$

$(124,119,129)$
F2

$(245,194,159)$

$(132,124,139)$

Table 5 Without Rayleigh scattering, the RGB (red, green, blue) triplets employed in the generation of the swatches presented in Fig. 7. Each RGB triplet is computed using the modelled reflectance data for the baseline skin specimen with and without a subcutaneous vein considering $\mathrm{CIE}$ standard illuminants D50, D65, A, and F2, as described in Sec. 4.4.

D50

Skin

Skin + vein
D65

$(237,206,168)$

$(103,77,72)$
A

$(248,200,171)$

$(111,74,72)$
F2

$(251,210,170)$

$(105,79,75)$ presented in Table 5 exceeds in magnitude its green and blue counterparts.

\subsection{Experiment Set \#2}

Swatches belonging to our second set of experimental results are presented in Fig. 8. They show that the role of Rayleigh scattering in the appearance of a skin specimen with a subcutaneous vein is consistent when considering different levels of melanin content and dermal blood content.

\subsection{Experiment Set \#3}

Since our results indicate that Rayleigh scattering in the papillary dermis contributes to the appearance of veins, let us examine the effect of varying the values of parameters that have an impact on the amount of Rayleigh scattering occurring in the papillary dermis.

The results presented in Fig. 9 show the effect of varying the radius of the fibrils in the papillary dermis on the reflectance of skin specimens with and without a subcutaneous vein. For the skin specimens without a subcutaneous vein [Fig. 9(a)], we can see that a higher fibril radius corresponds to a lower reflectance, except in the region near $400 \mathrm{~nm}$. For the skin specimen with a subcutaneous vein [Fig. 9(b)], a higher fibril radius corresponds to a higher reflectance across the visible spectrum. We can also observe in Fig. 9(b) that the difference between the reflectance of the baseline skin specimen $(r=40 \mathrm{~nm})$ and the reflectance of a skin specimen with a higher fibril radius $(r=50$ and $60 \mathrm{~nm})$ is the greatest in the middle of the visible spectrum, a region corresponding to what a human observer would perceive as green color. In both subfigures [Figs. 9(a) and 9(b)], we can see that skin specimens with a small fibril radius $(r=20$ and $30 \mathrm{~nm})$ have reflectance curves that resemble the reflectance curves presented in Fig. 6(b), which correspond to the experiments considering the baseline skin specimen without any Rayleigh scattering.

Now, let us examine Fig. 10 to see how these observations translate to appearance. In particular, when examining the appearance of the skin specimens with a subcutaneous vein, we can see that decreasing the fibril radius below the baseline value darkens the appearance of the vein and provides a 


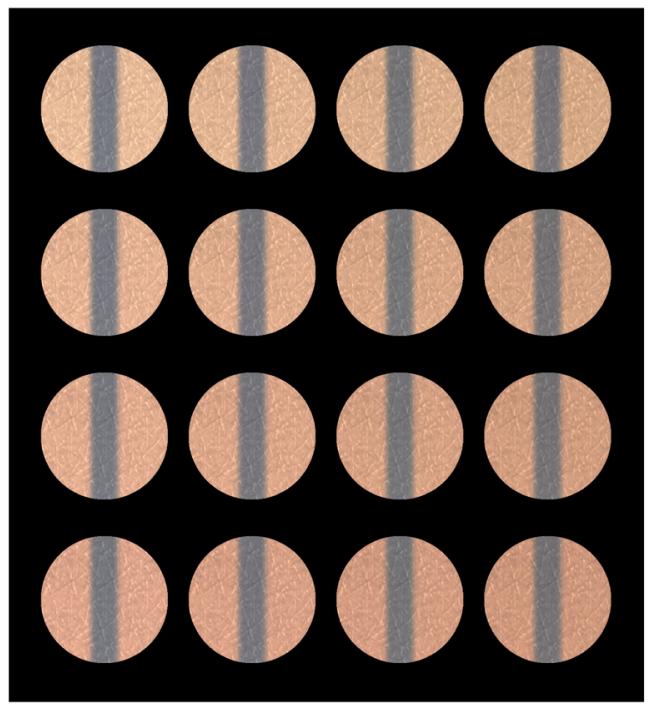

(a)

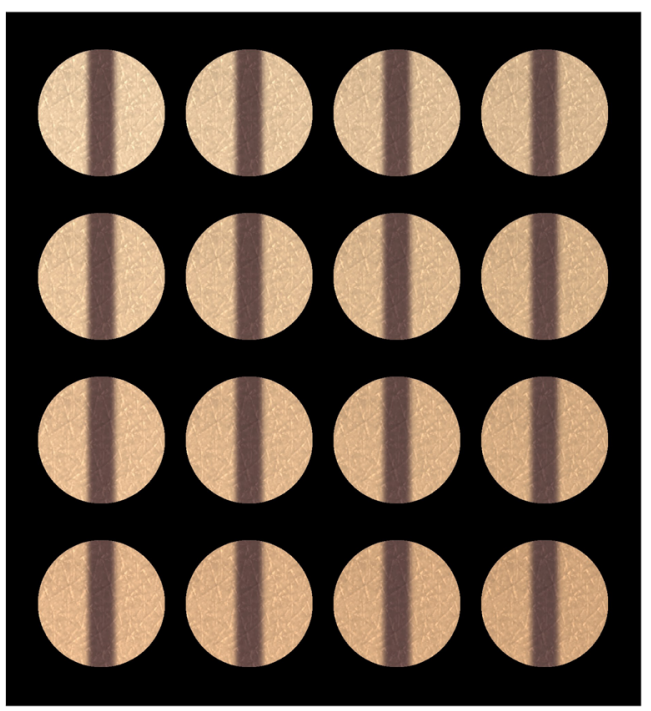

(b)

Fig. 8 Generated swatches depicting several skin specimens with a subcutaneous vein running beneath them considering the CIE standard illuminant D65. Distinct skin specimens were obtained by multiplying melanin and blood content (Table 2) by $f_{\mathrm{mel}}$ and $f_{\mathrm{bl}}$, respectively. Left to right: $f_{\mathrm{mel}}=1.0,1.5,2.0,2.5$. Top to bottom: $f_{\mathrm{bl}}=1.0,1.5,2.0,2.5$ : (a) with Rayleigh scattering and (b) without Rayleigh scattering. Note that the baseline skin specimen corresponds to $f_{\mathrm{mel}}=1.0$ and $f_{\mathrm{bl}}=1.0$.

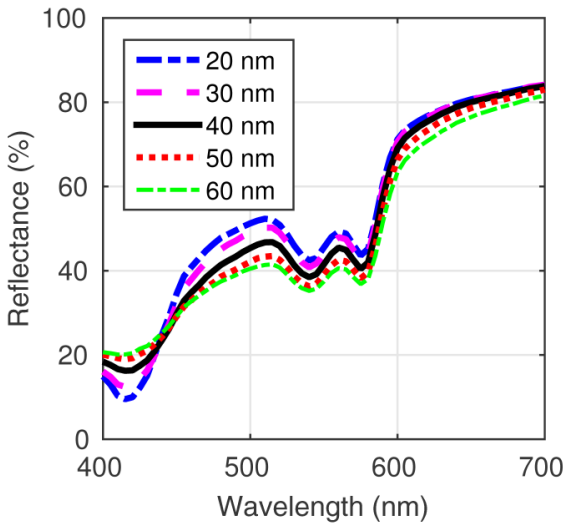

(a)

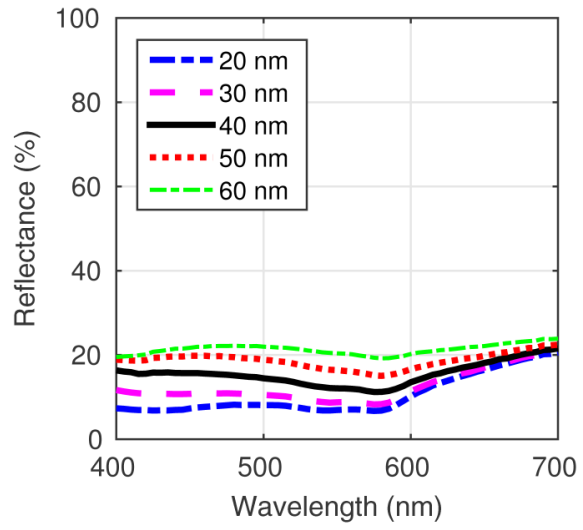

(b)

Fig. 9 Modelled reflectance curves for skin specimens considering varying radius values of the fibrils in the papillary dermis $(r)$ : (a) without a subcutaneous vein and (b) with a subcutaneous vein. Note that the baseline skin specimen has $r=40 \mathrm{~nm}$.

brownish-red or purple coloration. Alternatively, increasing the fibril radius above the baseline value results in a more greenish appearance.

We also performed experiments in which we varied the thickness of the papillary dermis. In the corresponding reflectance curves, presented in Fig. 11, we can observe similar trends to the experiments in which we varied the fibril radius (Fig. 9). However, the changes in reflectance are lower in magnitude when varying the papillary dermis thickness given the parameter values that we have selected. Consequently, the corresponding appearance swatches in Fig. 12 show variations in coloration similar, albeit less prominent, to the results presented in Fig. 10.

Now, let us examine the combined effect of varying both the radius of the fibrils and the papillary dermis thickness in Fig. 13. When examining the swatches that consider Rayleigh scattering [Fig. 13(a)], we can see that increasing one parameter value while decreasing the other can lead to more subtle changes in the appearance of the specimen, while increasing or decreasing both parameter values simultaneously can lead to more drastic changes in its appearance. On the other hand, when examining the swatches generated with Rayleigh scattering disabled [Fig. 13(b)], we can verify that similar changes do not take place. This demonstrates that any change in the appearance of a skin specimen with a subcutaneous vein resulting from varying the values of fibril radius and papillary dermis thickness is primarily determined by the occurrence of Rayleigh scattering in this tissue.

\section{Discussion}

Experiment set \#1 demonstrates that Rayleigh scattering plays a pivotal role in the bluish appearance of veins. Experiment set \#2 shows that varying blood and melanin content within the range 


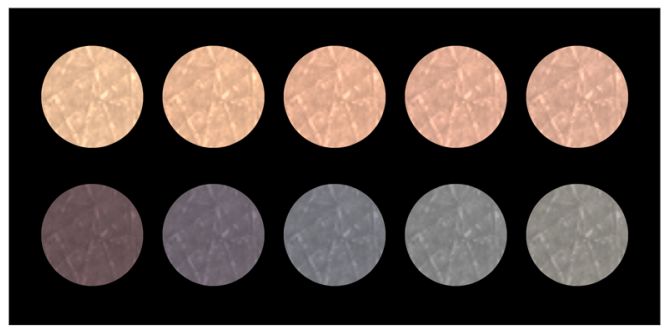

(a)

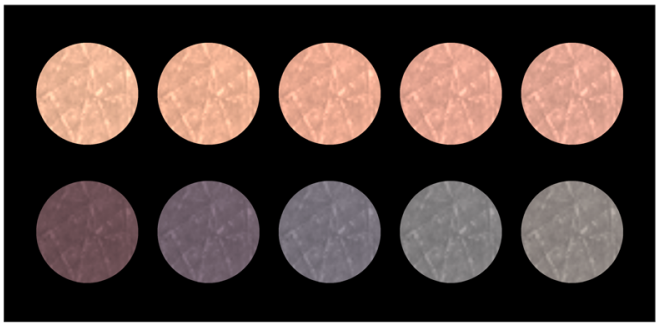

(c)

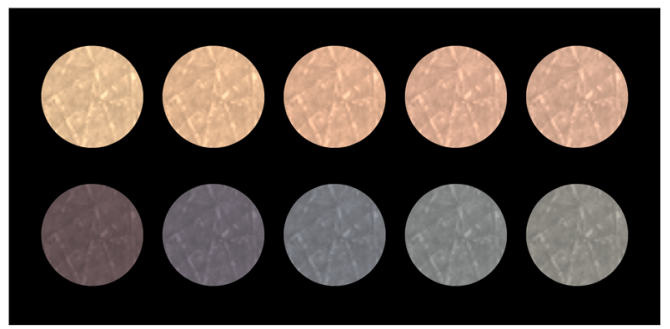

(b)

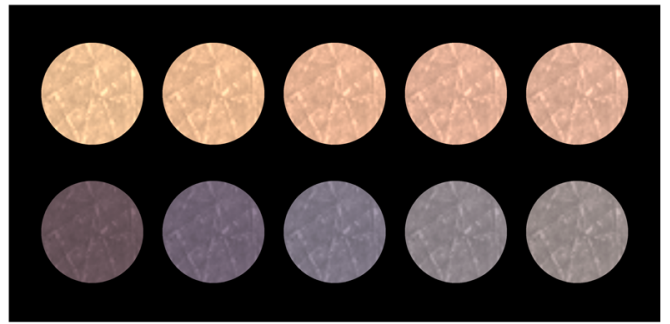

(d)

Fig. 10 Generated swatches depicting variations in skin appearance when modifying the radius of the fibrils in the papillary dermis $(r)$. For comparison purposes, we present swatches for specimens without (top) and with (bottom) a subcutaneous vein in each subfigure. For each subfigure, from left to right: $r=20,30,40,50,60 \mathrm{~nm}$. The swatches were generated considering CIE standard illuminants: (a) D50, (b) D65, (c) A, and (d) F2. Note that the baseline skin specimen has $r=40 \mathrm{~nm}$.

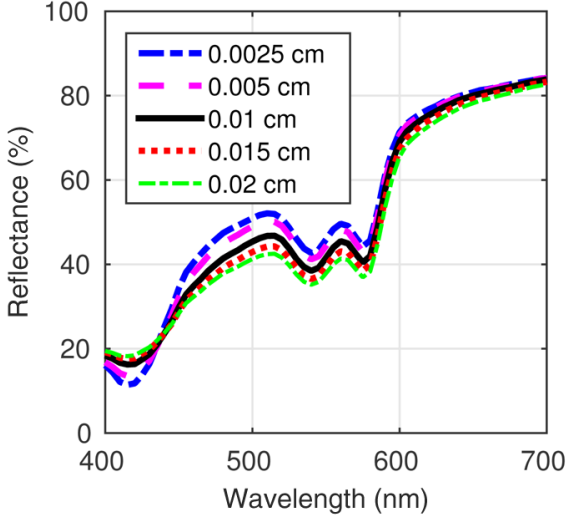

(a)

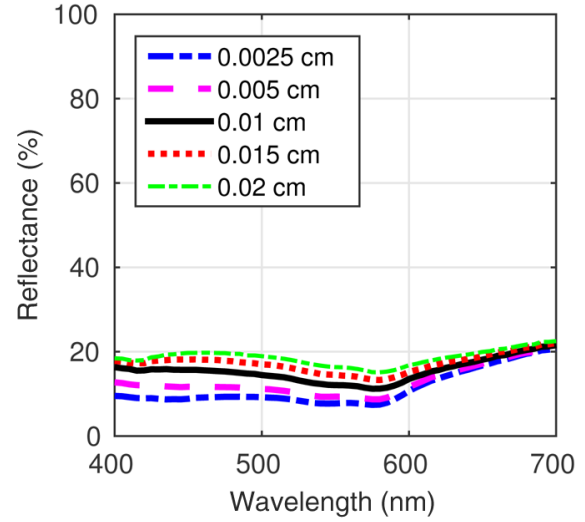

(b)

Fig. 11 Modelled reflectance curves for skin specimens considering varying papillary dermis thickness $\left(t_{\mathrm{pd}}\right)$ : (a) without a subcutaneous vein and (b) with a subcutaneous vein. Note that the baseline skin specimen has $t_{\mathrm{pd}}=0.02 \mathrm{~cm}$.

of physically plausible concentrations for a lightly pigmented specimen $^{64}$ does not affect the significance of the role of Rayleigh scattering in the appearance of veins. Experiment set \#3 demonstrates that the appearance of veins can depend on the morphological characteristics of the papillary dermis. Specifically, any parameter that contributes to the amount of Rayleigh scattering occurring in the papillary dermis (Sec. 4.3) could potentially affect the appearance of veins.

Although our results obtained in Experiment set \#1 cannot be directly compared with the in vivo measurements of Kienle et al. ${ }^{11}$ because of potential differences in experimental setup and specimen characterization, qualitative comparisons can be made. In particular, their measurements of the amount of light remitted by skin above a visible vein and the adjacent skin tissue shows that one should expect a lower reflectance when performing a measurement above a vein. Furthermore, when considering measurements performed at distinct wavelengths, their data shows that one should expect larger difference in reflectance for longer wavelengths (e.g., $633 \mathrm{~nm}$ and $700 \mathrm{~nm}$ ) than shorter wavelengths (e.g., $450 \mathrm{~nm}$ or $500 \mathrm{~nm}$ ) when comparing skin specimens with and without a subcutaneous vein. Both of these observations can also be verified in our results presented in Fig. 6(a).

It is also worth noting that we were able to quantitatively reproduce the bluish appearance of veins using standard CIE color space calculations (Table 4), which Kienle et al. ${ }^{11}$ were unable to achieve using their in vivo measurements. They suggested that this might be due to the fact that they employed only 


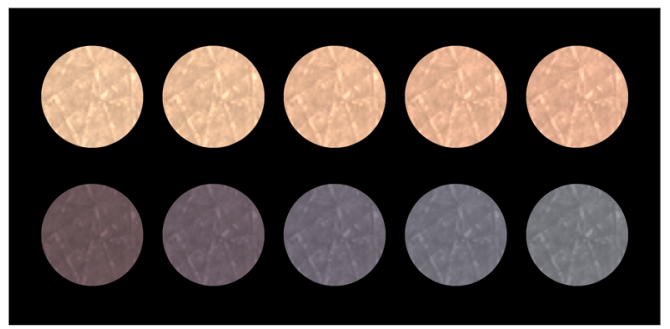

(a)

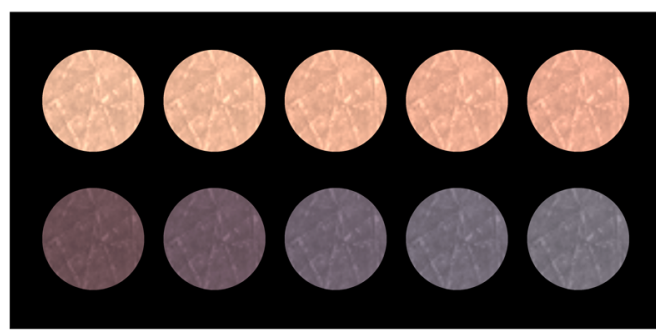

(c)

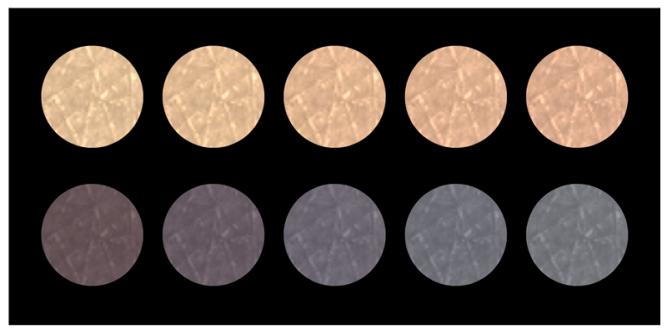

(b)

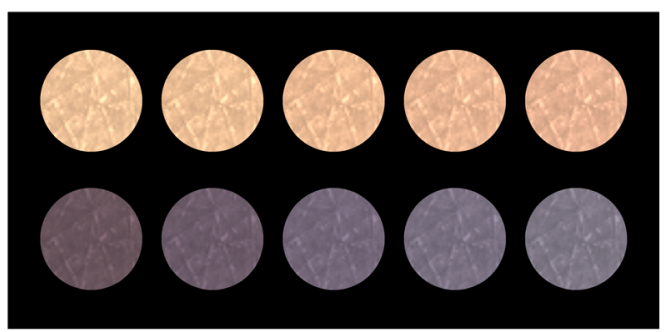

(d)

Fig. 12 Generated swatches depicting variations in skin appearance when modifying the thickness of the papillary dermis $\left(t_{\mathrm{pd}}\right)$. For comparison purposes, we present swatches for specimens without (top) and with (bottom) a subcutaneous vein in each subfigure. For each subfigure, from left to right: $t_{\mathrm{pd}}=0.0025$, $0.005,0.01,0.015,0.02 \mathrm{~cm}$. The swatches were generated considering CIE standard illuminants (a) D50, (b) D65, (c) A, and (d) F2. Note that the baseline skin specimen has $t_{\mathrm{pd}}=0.02 \mathrm{~cm}$.

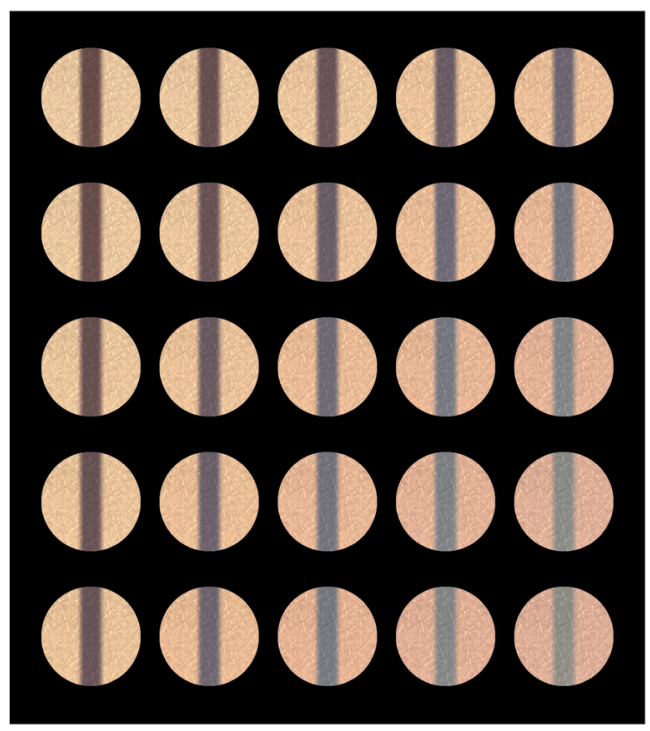

(a)

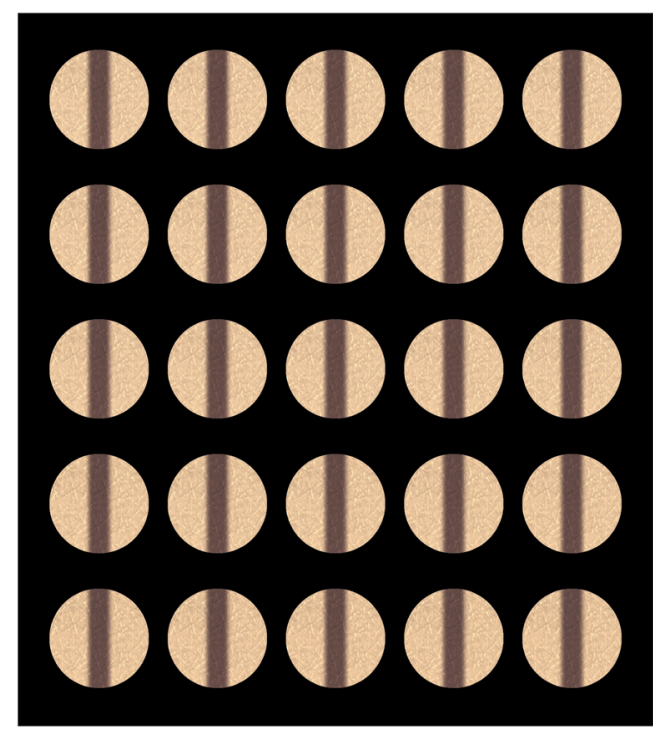

(b)

Fig. 13 Generated swatches depicting several skin specimens with a subcutaneous vein running beneath them considering the CIE standard illuminant D65. Distinct skin specimens were obtained by varying the radius of the fibrils in the papillary dermis $(r)$ and the papillary dermis thickness $\left(t_{\mathrm{pd}}\right)$. Left to right: $r=20,30,40,50,60 \mathrm{~nm}$. Top to bottom: $t_{\mathrm{pd}}=0.00250 .0025,0.005,0.01,0.015$, $0.02 \mathrm{~cm}$ : (a) with Rayleigh scattering and (b) without Rayleigh scattering. Note that the baseline skin specimen has $r=40 \mathrm{~nm}$ and $t_{\mathrm{pd}}=0.02 \mathrm{~cm}$.

five reflectance values obtained at close spectral locations. We, on the other hand, considered reflectance values sampled at 61 wavelengths at 5-nm intervals.

Experiment set \#3 also indicates an interesting feature of the fibrils in the papillary dermis. In particular, measurements by Arao et al. ${ }^{7}$ show that reticulin fibrils in the papillary dermis have a radius in the range $20-25 \mathrm{~nm}$, whereas the collagen fibrils in the papillary dermis have a radius in the range $40-60 \mathrm{~nm}$. Our experiments considering fibrils with $20 \mathrm{~nm}$ radius [Figs. 10 and 13(a)] result in veins with a brownish-red to purple appearance. In fact, this observation is similar to the corresponding observation derived from the experiments in which we did not consider Rayleigh scattering [Fig. 13(b)]. Since we fixed the volume fraction of the papillary dermis occupied by fibrils $\left(v_{\mathrm{f}}\right)$ and, physiologically, reticulin fibrils only make up a fine layer of the papillary dermis, ${ }^{7}$ Rayleigh scattering caused by reticulin 
fibrils located in the papillary dermis is unlikely to significantly contribute to the bluish appearance of veins. Therefore, our findings indicate that the Rayleigh scattering (occurring in the papillary dermis) that contributes to the bluish appearance of veins is primarily elicited by collagen fibrils.

In the following subsections, we discuss additional considerations regarding our simulation choices and results.

\subsection{Intermediate Tissues}

In our experiments, we do not fully consider the attenuating properties of the vein wall. We also do not consider a hypodermis between the skin and the vein. In the human body, if a vein is deep enough, then scattering in the hypodermis ensures that the vein is not visible. Accordingly, it is possible that a less visually prominent vein may have a thin hypodermal layer between itself and the skin. Although these intermediate tissues may impact the reflectance of skin in general, it is unlikely that they can be the primary source of a vein's bluish appearance given the optical properties of their main constituents. In the remainder of this subsection, we examine this claim in more detail.

The weak absorption of visible light in these tissues is dominated by hemoglobin. ${ }^{45-47}$ Since hemoglobin is abundant in the subcutaneous vein, these tissues should have a minor impact on the overall absorption. However, if we were to consider the effect of hemoglobin in these tissues, it should be similar to increasing the hemoglobin concentration in the reticular dermis. As we can see in Fig. 8, this does not have a significant effect on the bluish appearance of the vein.

There is also a possibility that small heterogeneous structures in these intermediate tissues produce Rayleigh scattering. However, our experiments indicate that Rayleigh scattering in the skin is strong enough to elicit the bluish appearance of veins without the consideration of these tissues. This is corroborated by the observation that cyanotic fingertips would not appear bluish if Rayleigh scattering in the papillary dermis was not sufficient to prevent blue light from reaching the reticular dermis. ${ }^{12}$ Since Rayleigh scattering in the skin occurs before light reaches the hypodermis or vein wall, any additional Rayleigh scattering in these subcutaneous tissues could only increase the, already sufficient, remittance of blue light.

\subsection{Vein Appearance}

In this paper, we have been focusing on the bluish appearance of veins. As demonstrated by experiment set \#3 (Figs. 9-13), a subcutaneous vein may also appear to be subjectively more green, purple, or gray than blue when considering different physiological states and illumination conditions. These findings indicate that Rayleigh scattering occurring in the papillary dermis plays an important role in the appearance of any visible subcutaneous vein, even though its contribution may not always result in a bluish appearance.

\subsection{Pigmentation Level}

We have chosen to focus on lightly pigmented specimens in our investigation since melanin absorbs light in the blue region significantly more than in other regions [Fig. 2(a)]. This indicates that additional melanin in the epidermis would increase the absorption of blue light before it reaches the papillary dermis, which would inhibit our ability to examine the effects of Rayleigh scattering caused by the collagen fibrils. We remark, however, that examining the appearance of veins for moderately and highly pigmented specimens may be a topic of interest for future investigation (Sec. 7).

\subsection{Venous Blood Characterization}

We performed several additional experiments to assess the effect of varying the venous blood characterization on the appearance of a skin specimen with a subcutaneous vein. When experimenting with various oxygen saturation levels, we observed that variations within a typical physiological range did not have a noticeable impact on coloration. However, arterial oxygenation levels (e.g., 97\%) resulted in a skin specimen with a subcutaneous vein reflecting more red light, which, in turn, elicited a purple appearance. Alternatively, extremely low levels of oxygenation levels (e.g., 0\%) resulted in less reflected red light, which, in turn, elicited a more dominant blue coloration. Since this behavior is consistent with observations by Kienle et al., ${ }^{11}$ we excluded these results for the sake of conciseness. We also explored different distributions of red blood cell orientations in the flowing venous blood, corresponding to different shear rates. This did not noticeably affect the resulting coloration.

\subsection{Hypodermis Reflectance}

In Sec. 3.2, we mentioned that in our simulations, visible light reaching the hypodermis is diffusely reflected back to the reticular dermis. We also noted that this simulation choice was based on reported morphological and optical characteristics of white adipose tissues (Sec. 2.3) and it is consistent with simulation strategies employed by related works. ${ }^{10,52}$ We remark that most of our observations presented in this work (Secs. 5 and 6) correspond to a skin specimen with a subcutaneous vein beneath it. Moreover, the direct observation made for a skin specimen with a hypodermis beneath it (Sec. 5.1) focuses on a feature of skin reflectance curve (Fig. 6) in the blue region and emphasizes the light that does not reach the hypodermis. Hence, the outcomes of our investigation are not impaired by the simulation choice outlined above.

\subsection{Vein Transmittance}

In Sec. 3.3, we mentioned that our simulations discard light rays that are transmitted by the vein. This strategy was motivated by the low probability of light being transmitted by the vein $\left(p_{\text {vein }}^{t}\right)$, depicted in the curve presented in Fig. 14(a). This curve was calculated using the same approach employed for precomputing $p_{\text {vein }}^{r}$ (Fig. 4), described in Sec. 3.4. Now, let us consider the worst-case scenario, where $100 \%$ of light transmitted by the vein would be diffusely remitted by the tissue below. In this case, the amount of light transmitted by the vein and then subsequently remitted by it would be given by $\left(p_{\text {vein }}^{t}\right)^{2}$, which is presented in Fig. 14(b). We note that the maximum value on this curve is $0.53 \%$ at $700 \mathrm{~nm}$. This would represent a negligible contribution to the overall reflectance of a skin specimen with a subcutaneous vein (Fig. 6), notably when compared with the probability of light being reflected by the vein without considering a remission of light by tissues below the vein (Fig. 4). Hence, the decision of discarding the light rays that might be transmitted by the vein did not affect the observations derived from our in silico experiments, especially considering 


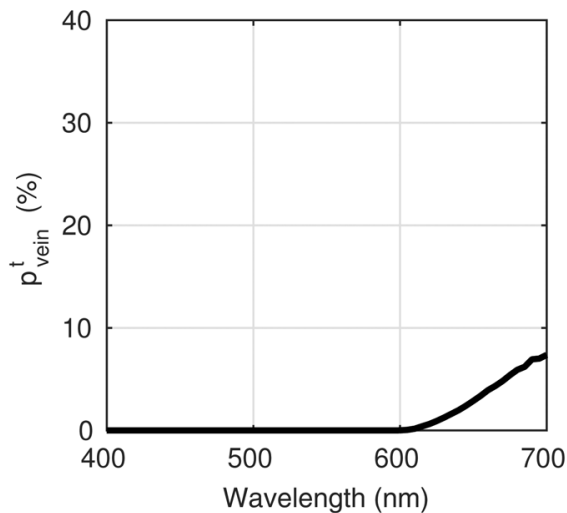

(a)

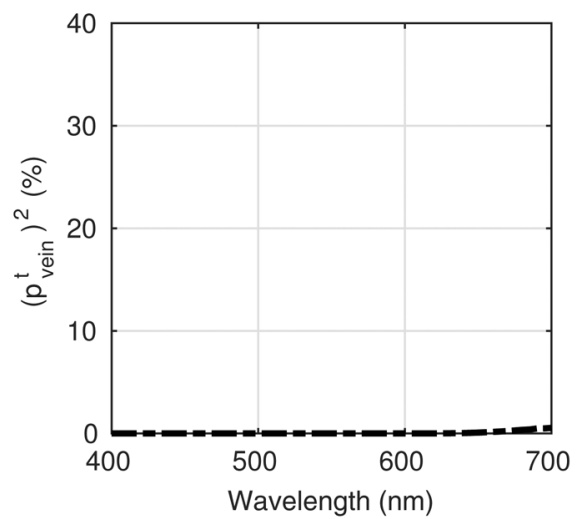

(b)

Fig. 14 Computed transmittance data for the subcutaneous vein specimen employed in our experiments. (a) Probability of light being transmitted by the vein $\left(p_{\text {vein }}^{t}\right)$. (b) Squared probability of light being transmitted by the vein $\left[\left(p_{\text {vein }}^{t}\right)^{2}\right]$. The values for $p_{\text {vein }}^{t}$ were computed using an analogous procedure to the precomputation outlined in Sec. 3.4, considering the blood characterization data presented in Table 3.

that $\left(p_{\text {vein }}^{t}\right)^{2}$ values are even smaller for other wavelengths [Fig. 14(b)].

\section{Conclusion and Future Work}

The in silico experimental results presented in this paper support the hypothesis that Rayleigh scattering caused by collagen fibrils found in the papillary dermis can play a pivotal role in the bluish appearance of veins. In particular, Rayleigh scattering occurring in the papillary dermis remits blue light from the skin before it can reach the blood flowing through the subcutaneous vein. The blood flowing through the vein then moderately absorbs the red light that reaches it. The net effect of these two optical processes is the bluish appearance of veins. In addition, these results show that distinct vein colorations, such as gray or greenish, may result from different amounts of Rayleigh scattering associated with variations in the morphological characteristics of the papillary dermis, notably its thickness and the radius of its collagen fibrils. Finally, our experimental results indicate that Rayleigh scattering caused by reticulin fibrils located in the papillary dermis is not a significant contributor to the bluish appearance of veins.

As with any in silico investigation, the outcomes of our research require confirmation from in situ experiments. We remark, however, that our in silico experiments were performed using first-principles models, whose predictive capabilities have been extensively evaluated in previous works, ${ }^{14-16}$ and considering measured biophysical data provided in the scientific literature. Hence, we are confident that our findings can be corroborated by in situ experiments as new technologies that allow measurements to be performed within the papillary dermis under in vivo conditions become available.

There are many possibilities for future work. For example, it may be useful to evaluate the impact of the masking effect of melanosomes on the appearance of subcutaneous veins in moderately and darkly pigmented skin specimens, in a similar manner to the work of Baranoski et al. ${ }^{113}$ regarding cyanosis. In addition to strengthening the current understanding of fundamental optical mechanisms occurring within the skin, investigations within the realm of the work presented in this paper could aid in the design of more effective optical devices for the noninvasive detection of physiological changes within the skin and subcutaneous veins. These changes could include, for instance, variations in the concentration of blood-borne substances, such as bilirubin and methemoglobin, associated with the onset of potentially life-threatening conditions like hyperbilirubinemia and methemoglobinemia, respectively.

From a visual perspective, a more in-depth exploration of distinct skin specimen characterizations could enable the predictive reproduction of various skin tones caused by subcutaneous blood vessels. Additionally, placing the vein representation within the cutaneous tissues would allow us to simulate the appearance of enlarged cutaneous vessels. These advancements could contribute to the improvement of computer-generated representations of skin, which, in turn, would enhance the effectiveness of scientific, educational, and entertainment applications requiring realistic visualizations of the human body.

\section{Disclosures}

The authors have no relevant financial interests in the manuscript and no other potential conflicts of interest to disclose.

\section{Acknowledgments}

This work was supported by the Natural Sciences and Engineering Research Council of Canada (NSERC-Discovery Grant 237337).

\section{References}

1. J. W. Strutt, "On the scattering of light by small particles," Philos. Mag. 41, 447-454 (1871).

2. J. W. Strutt, "On the transmission of light through an atmosphere containing many small particles in suspension, and on the origin of the blue of the sky," Philos. Mag. 47, 375-384 (1899).

3. E. A. Edwards and S. Q. Duntley, "The pigments and color of living human skin," Am. J. Anat. 65(1), 1-33 (1939).

4. R. R. Anderson and J. A. Parrish, "The optics of human skin," J. Invest. Dermatol. 77(1), 13-19 (1981).

5. G. Findlay, "Blue skin," Br. J. Dermatol. 83(1), 127-134 (1970).

6. A. Oettlé, "The blue scrotum of the vervet monkey," S. Afr. J. Med. Sci. 23, 225-230 (1958). 
7. H. Arao et al., "Morphological characteristics of the dermal papillae in the development of pressure sores," J. Tissues Viability 8(3), 17-23 (1998).

8. I. Saidi, S. Jacques, and F. Tittel, "Mie and Rayleigh modeling of visible-light scattering in neonatal skin," Appl. Opt. 34, 7410-7418 (1995).

9. S. L. Jacques, "Origins of tissue optical properties in the UVA, visible, and NIR regions," in OSA TOPS on Advances in Optical Imaging and Photon Migration, Vol. 2, pp. 364-371 (1996).

10. S. Cotton and E. Claridge, "Developing a predictive model of skin colouring," Proc. SPIE 2708, 814-825 (1996).

11. A. Kienle et al., "Why do veins appear blue? A new look at an old question," Appl. Opt. 35(7), 1151-1160 (1996).

12. G. V. G. Baranoski, S. R. Van Leeuwen, and T. F. Chen, "Elucidating the biophysical processes responsible for the chromatic attributes of peripheral cyanosis," in 39th Annual Int. Conf. of the IEEE Engineering in Medicine and Biology Society (EMBC), Jeju Island, Korea, pp. 90-95 (2017).

13. S. R. Van Leeuwen and G. V. G. Baranoski, "Identifying the optical phenomena responsible for the blue appearance of veins," Proc. SPIE 10367, 103670F (2017).

14. T. F. Chen et al., "Hyperspectral modeling of skin appearance," $A C M$ Trans. Graphics 31(3), 1-14 (2015).

15. D. Yim et al., "A cell-based light interaction model for human blood," Comput. Graphics Forum 31(2pt4), 845-854 (2012).

16. S. R. Van Leeuwen, G. V. G. Baranoski, and B. W. Kimmel, "Revisiting the CLBlood model: Formulation enhancements and online deployment," Technical Report CS-2017-01, University of Waterloo, School of Computer Science (2017).

17. T. Lister, P. A. Wright, and P. H. Chappell, "Optical properties of human skin," J. Biomed. Opt. 17(9), 090901 (2012).

18. S. L. Jacques, "Optical absorption of melanin," Technical Report, Oregon Medical Laser Center, Portland, Oregon (2001).

19. S. A. Prahl, "PhotochemCAD spectra by category," Technical Report, Oregon Medical Laser Center, Portland, Oregon (2001).

20. S. A. Prahl, "Optical absorption of hemoglobin," Technical Report, Oregon Medical Laser Center, Portland, Oregon (1999).

21. L. L. Randeberg et al., "Methemoglobin formation during laser induced photothermolysis of vascular skin lesions," Lasers Surg. Med. 34(5), 414-419 (2004).

22. O. Siggaard-Andersen, B. Nørgaard-Pedersen, and J. Rem, "Hemoglobin pigments. spectrophotometric determination of oxy-, carboxy-, met-, and sulfhemoglobin in capillary blood," Clin. Chim. Acta 42(1), 85-100 (1972).

23. I. H. Yarynovska and A. I. Bilyi, "Absorption spectra of sulfhemoglobin derivatives of human blood," Proc. SPIE 6094, 60940G (2006).

24. K. M. V. de Graaff, Human Anatomy, 4th ed., W. C. Brown Publishers, Dubuque, Iowa (1995).

25. R. R. Anderson and J. A. Parrish, "Optical properties of human skin," in The Science of Photomedicine, J. D. Regan and J. A. Parrish, Eds., 147-194, Plenun Press, New York (1982).

26. M. A. Pathak, "Functions of melanin and protection by melanin," in Melanin: Its Role in Human Photoprotection, M. R. C. L. Zeise and T. B. Fitzpatrick, Eds., pp. 125-134, Valdenmar Publishing Co., Overland Park, Kansas (1995).

27. W. A. G. Bruls and J. C. Van Der Leun, "Forward scattering properties of human epidermal layers," Photochem. Photobiol. 40(2), 231-242 (1984).

28. M. van Gemert et al., "Skin optics," IEEE Trans. Biomed. Eng. 36(12), 1146-1154 (1989).

29. N. Kollias et al., "Photoprotection by melanin," J. Photochem. Photobiol. B 9(2), 135-160 (1991).

30. R. L. Olson, J. Gaylor, and M. A. Everett, "Skin color, melanin, and erythema," Arch. Dermatol. 108(4), 541-544 (1973).

31. G. Szabo et al., "Racial differences in the fate of melanosomes in human epidermis," Nature 222, 1081-1082 (1969).

32. J. A. Jacquez et al., "Spectral reflectance of human skin in the region

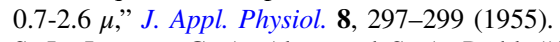

33. S. L. Jacques, C. A. Alter, and S. A. Prahl, "Angular dependence of HeNe laser light scattering by human dermis," Lasers Life Sci. 1, 309-333 (1987).

34. M. Meinke et al., "Optical properties of platelets and blood plasma and their influence on the optical behavior of whole blood in the visible to near infrared wavelength range," J. Biomed. Opt. 12(1), 014024 (2007).

35. M. Kinnunen et al., "Effect of the size and shape of a red blood cell on elastic light scattering properties at the single-cell level," Biomed. Opt. Express 2(7), 1803-1814 (2011).

36. L.-G. Lindberg and P. A. Oberg, "Optical properties of blood in motion,” Opt. Eng. 32(2), 253-257 (1993).

37. P. Agache and S. Diridollou, "Subcutis histopathology," in Measuring the Skin, P. Agache and P. Humbert, Eds., pp. 401-409, SpringerVerlag, Berlin (2004).

38. A. N. Bashkatov et al., "Optical properties of human skin, subcutaneous and mucous tissues in the wavelength range from 400 to $2000 \mathrm{~nm}$," J. Phys. D: Appl. Phys. 38, 2543-2555 (2005).

39. J. Schmitt, A. Knüttel, and R. Bonner, "Measurement of optical properties of biological tissues by low-cogerence reflectometry," Appl. Opt. 32(30), 6032-6041 (1993).

40. K. P. Nielsen et al., "Reflectance spectra of pigmented and nonpigmented skin in the UV spectral region," Photochem. Photobiol. 80, 450-455 (2004).

41. G. B. Altshuler, R. R. Anderson, and D. Manstein, "Method and apparatus for the selective targeting of lipid-rich tissues," U.S. Patent 6,605,080 (2003).

42. R. M. Pope and E. S. Fry, "Absorption spectrum (380-700 nm) of pure water. II. Integrating cavity measurements," Appl. Opt. 36, 8710-8723 (1997).

43. K. F. Palmer and D. Williams, "Optical properties of water in the near infrared," J. Opt. Soc. Am. 64, 1107-1110 (1974).

44. U. Dinish et al., "Diffuse optical spectroscopy and imaging to detect and quantify adipose tissue browning," Sci. Rep. 7, 41357 (2017).

45. M. F. Yang, V. V. Tuchin, and A. N. Yaroslavsky, "Principles of lightskin interactions," in Light-Based Therapies for Skin of Color, E. D. Baron, Ed., pp. 1-44, Springer-Verlag, London (2009).

46. J. Švejcar et al., "Content of collagen, elastin, and water in walls of the internal saphenous vein in man," Circ. Res. 11(2), 296-300 (1962).

47. A. N. Bashkatov, E. A. Genina, and V. V. Tuchin, "Optical properties of skin, subcutaneous, and muscle tissues: a review," J. Innovative Opt. Health Sci. 4(01), 9-38 (2011).

48. W. L. Butler, "Absorption spectroscopy in vivo theory and application," Ann. Rev. Plant Physiol. 15(1), 451-460 (1964).

49. P. Latimer, "A wave-optics effect which enhances light absorption by chlorophyll in vivo," Photochem. Photobiol. 40(2), 193-199 (1984).

50. Natural Phenomena Simulation Group (NPSG), "Human skin data," http://www.npsg.uwaterloo.ca/data/skin.php (2017).

51. Natural Phenomena Simulation Group (NPSG), "Human blood data," http://www.npsg.uwaterloo.ca/data/blood.php (2017).

52. M. Doi and S. Tominaga, "Spectral estimation of human skin color using the Kubelka-Munk theory," Proc. SPIE 5008, 221-228 (2003).

53. G. V. G. Baranoski, J. G. Rokne, and G. Xu, "Virtual spectrophotometric measurements for biologically and physically based rendering," Visual Comput. 17(8), 506-518 (2001).

54. B. D. Fornage and J.-L. Deshayes, "Ultrasound of normal skin," J. Clin. Ultrasound 14(8), 619-622 (1986).

55. P. S. Talreja et al., "Visualization of the lipid barrier and measurement of lipid pathlength in human stratum corneum," AAPS PharmSci. 3(2), 48-56 (2001).

56. N. Magnenat-Thalmann et al., "A computational skin model: fold and wrinkle formation," IEEE Trans. Inf. Technol. Biomed. 6(4), 317-323 (2002).

57. G. Plewig et al., "Thickness of the corneocytes," in Stratum Corneum, R. Marks and G. Plewig, Eds., pp. 171-174, Springer-Verlag, Berlin (1983).

58. K. Robertson and J. L. Rees, "Variation in epidermal morphology in human skin at different body sites as measured by reflectance confocal microscopy," Acta Derm.-Venereol. 90, 368-373 (2010).

59. P. Agache, "Metrology of the stratum corneum," in Measuring the Skin, pp. 101-111, Springer-Verlag, Berlin (2004).

60. B. Querleux, L. Darrasse, and J. Bittoun, "Magnetice resonance imaging of human skin in vivo," in Bioengineering of the Skin Skin Imaging and Analysis, K. Wilhelm et al., Eds., pp. 99-109, CRC Press, Boca Raton, Florida (2007). 
61. K. Robertson and J. L. Rees, "Variation in epidermal morphology in human skin at different body sites as measured by reflectance confocal microscopy," Acta Derm.-Venereol. 90(4), 368-373 (2010).

62. H. Shimizu, Shimizu's Textbook of Dermatology, Hokkaido University Press, Sapporo, Japan (2007).

63. A. Caduff, M. S. Talary, and P. Zakharov, "Cutaneous blood perfusion as a perturbing factor for noninvasive glucose monitoring," Diabetes Technol. Ther. 12(1), 1-9 (2010).

64. T. Lister, "Simulating the color of port wine stain skin," PhD Thesis, University of Southampton (2013).

65. A. J. Thody et al., "Pheomelanin as well as eumelanin is present in human epidermis," J. Invest. Dermatol. 97, 340-344 (1991).

66. A. Hennessy et al., "Eumelanin and pheomelanin concentrations in human epidermis before and after UVB irradiation," Pigm. Cell Res. 18, 220-223 (2005).

67. M. L. Noll and J. F. Byers, "Usefulness of measures of $\mathrm{Svo}_{2}, \mathrm{Spo}_{2}$, vital signs, and derived dual oximetry parameters as indicators of arterial blood gas variables during weaning of cardiac surgery patients from mechanical ventilation," Heart Lung 24(3), 220-227 (1995).

68. A. T. Lovell et al., "Determination of the transport scattering coefficient of red blood cells," Proc. SPIE 3597, 175-182 (1999).

69. R. Flewelling, "Noninvasive optical monitoring," in The Biomedical Engineering Handbook, J. D. Bronzino, Ed., 2nd ed., CRC Press LLC, Boca Raton, Florida (2000).

70. C. S. E. S. Haymond, R. Cariappa, and M. G. Scott, "Laboratory assessment of oxygenation in methemoglobinemia," Clin. Chem. 51(2), 434-444 (2005).

71. A. J. Cunnington et al., "Carboxyhemoglobin levels in Kenyan children with plasmodium falciparum malaria," Am. J. Trop. Med. Hyg. 71(1), 43-47 (2004).

72. B. Rolinski et al., "Total bilirubin measurement by photometry on a blood gas analyzer: potential for use in neonatal testing at the point of care," Clin. Chem. 47(10), 1845-1847 (2001).

73. R. Lee et al., "The detection of carotenoid pigments in human skin," J. Invest. Dermatol. 64, 175-177 (1975).

74. P. Agache, "Main skin biological constants," in Measuring the Skin, P. Agache and P. Humbert, Eds., pp. 727-746, Springer-Berlag, Berlin, Germany (2004).

75. N. Nakagawa, M. Matsumoto, and S. Sakai, "In vivo measurement of the water content in the dermis by confocal Raman spectroscopy," Skin Res. Technol. 16(2), 137-141 (2010).

76. J. A. Viator et al., "A comparative study of photoacoustic and reflectance methods for determination of epidermal melanin content," J. Invest. Dermatol. 122, 1432-1439 (2004).

77. M. L. Williams, M. Hincenbergs, and K. A. Holbrook, "Skin lipid content during early fetal development," J. Invest. Dermatol. 91, 263-268 (1988).

78. C. A. Squier, P. Cox, and P. W. Wertz, "Lipid content and water permeability of skin and oral mucosa," J. Invest. Dermatol. 96(1), 123-126 (1991).

79. A. E. Cerussi et al., "Sources of absorption and scattering contrast for near-infrared optical mammography," Acad. Radiol. 8, 211-218 (2001).

80. E. Fuchs, "Keratins and the skin," Annu. Rev. Cell Dev. Biol. 11(1), 123-154 (1995).

81. D. J. Gawkrodger, Dermatology An Illustrated Colour Text, Churchill Livingstone, Edinburgh, United Kingdom (2002).

82. A. R. Young, "Chromophores in human skin," Phys. Med. Biol. 42(5), 789-802 (1997).

83. J. S. Varcoe, Clinical Biochemistry: Techniques and Instrumentation: A Practical Course, World Scientific, Singapore (2001).

84. R. Flindt, Amazing Numbers in Biology, Springer, Berlin, Germany (2006).

85. G. J. Tearney et al., "Determination of the refractive index of highly scattering human tissue by optical coherence tomography," Opt. Lett. 20, 2258-2260 (1995).

86. B. L. Diffey, "A mathematical model for ultraviolet optics in skin," Phys. Med. Biol. 28(6), 647-657 (1983).

87. V. V. Tuchin, Tissue Optics: Light Scattering Methods and Instruments for Medical Diagnosis, SPIE Press, Bellingham, Washington (2007).
88. A. N. Bashkatov et al., "Optical properties of melanin in the skin and skinlike phantoms," Proc. SPIE 4162, 219-226 (2000).

89. X. Wang et al., "Group refractive index measurement of dry and hydrated type I collagen films using optical low-coherence reflectometry," J. Biomed. Opt. 1(2), 212-216 (1996).

90. S. Alaluf et al., "Ethnic variation in melanin content and composition in photoexposed and photoprotected human skin," Pigm. Cell Res. 15, 112-118 (2002).

91. I. M. Braverman, "The cutaneous microcirculation," J. Invest. Dermatol. Symp. Proc. 5(1), 3-9 (2000).

92. B. J. Bain et al., Dacie and Lewis Practical Haematology, 11th ed., Elsevier, Churchill, Livingstone (2012).

93. F. C. Mokken et al., "Differences in peripheral arterial and venous hemorheologic parameters," Ann. Hematol. 73(3), 135-137 (1996).

94. A. Kiray et al., "Anatomical evaluation of the superficial veins of the upper extremity as graft donor source in microvascular reconstructions: a cadaveric study," Acta Orthop. Traumatol. Turc. 47(6), 405-410 (2013).

95. M. Klarhöfer et al., "High-resolution blood flow velocity measurements in the human finger," Magn. Reson. Med. 45(4), 716-719 (2001).

96. T. G. Papaioannou and C. Stefanadis, "Vascular wall shear stress: basic principles and methods," Hell. J. Cardiol. 46(1), 9-15 (2005).

97. C. W. Yoxall and A. M. Weindling, "Measurement of venous oxyhaemoglobin saturation in the adult human forearm by near infrared spectroscopy with venous occlusion," Med. Biol. Eng. Comput. 35(4), 331-336 (1997).

98. S. Haymond et al., "Laboratory assessment of oxygenation in methemoglobinemia," Clin. Chem. 51(2), 434-444 (2005).

99. A. Roggan et al., "The optical properties of biological tissue in the near infrared wavelength range," in Laser-induced Interstitial Therapy, pp. 10-44, SPIE Press, Bellingham, Washington (1995).

100. E. J. McCartney, Optics of the Atmosphere: Scattering by Molecules and Particles, John Wiley \& Sons, New York (1976).

101. D. Arifler et al., "Light scattering from collagen fiber networks: microoptical properties of normal and neoplastic stroma," Biophys. J. 92, 3260-3274 (2007).

102. C. Borren and D. Huffman, Absoprtion and Scattering by Small Particles, Wiley, New York (1983).

103. C. Bohren, "Scattering by particles," Chapter 6 in Handbook of Optics, Vol. 1, Fundamentals, Techniques, and Design, M. Bass et al., Eds., pp. 6.1-6.21, Optical Society of America, McGraw-Hill, Inc., New York (1995).

104. A. Krishnaswamy, "BioSpec: a biophysically-based spectral model of light interaction with human skin," Master's Thesis, School of Computer Science, University of Waterloo, Waterloo, Ontario, Canada (2005).

105. S. A. Prahl, Light Transport in Tissue, PhD Thesis, University of Texas at Austin (1988).

106. R. W. G. Hunt, Measuring Colour, 2nd ed., Ellis Horwood Limited, Chichester, England (1991).

107. G. V. G. Baranoski and A. Krishnaswamy, Light \& Skin Interactions: Simulations for Computer Graphics Applications, Morgan Kaufmann/ Elsevier, Burlington, Massachusetts (2010).

108. E. H. Land and J. J. McCann, "Lightness and retinex theory," J. Opt. Soc. Am. 61(1), 1-11 (1971).

109. Natural Phenomena Simulation Group (NPSG), "Run HyLIoS online," http://www.npsg.uwaterloo.ca/models/hylios.php (2017).

110. Natural Phenomena Simulation Group (NPSG), "Run CLBlood online," http://www.npsg.uwaterloo.ca/models/clblood.php (2017).

111. G. V. G. Baranoski et al., "Rapid dissemination of light transport models on the web," IEEE Comput. Graphics Appl. 32, 10-15 (2012).

112. Natural Phenomena Simulation Group (NPSG), "NPSGD framework," http://www.npsg.uwaterloo.ca/models/npsgd_software.php (2017).

113. G. V. G. Baranoski, S. R. Van Leeuwen, and T. F. Chen, "On the detection of peripheral cyanosis in individuals with distinct levels of cutaneous pigmentation," in 39th Annual Int. Conf. of the IEEE Engineering in Medicine and Biology Society (EMBC), Jeju Island, Korea, pp. 4260-4264 (2017). 
Spencer R. Van Leeuwen is currently pursuing his master's degree in computer science at the David R. Cheriton School of Computer Science at the University of Waterloo (Canada). He holds a bachelor of mathematics degree in computer science and combinatorics and optimization with a minor in music from the same institution. He is also a member of the Natural Phenomena Simulation Group at the University of Waterloo.
Gladimir V. G. Baranoski obtained his PhD in computer science at the University of Calgary (Canada) in 1998. Currently, he is a faculty member of the David R. Cheriton School of Computer Science at the University of Waterloo (Canada), where he established the Natural Phenomena Simulation Group. His research interests primarily include the predictive simulation of light interactions with natural materials aimed at interdisciplinary applications. He is a member of SPIE. 